\title{
Optimal design for inspection and maintenance policy based on the CCC chart
}

\author{
LING-YAU CHAN \\ Department of Industrial and Manufacturing Systems Engineering, The University of \\ Hong Kong, Pokfulam Road, Hong Kong. e-mail: plychan@hku.hk \\ SHAOMIN WU \\ School of Applied Science, Cranfield UNiversity, \\ Cranfield, Bedfordshire, MK43 0AL, U. K. e-mail: shaomin.wu@cranfield.ac.uk
}

\begin{abstract}
In the paper, the concept of cumulative count of conforming chart (CCC chart) is applied in inspection and maintenance planning for systems where minor inspection, major inspection, minor maintenance and major maintenance are available. Several inspection and maintenance plans are defined and studied quantitatively. Analytic expressions of relevant statistics and their expectations are derived. These inspection and maintenance plans are optimized from an economic consideration.
\end{abstract}

Keywords. Inspection, maintenance, manufacturing, fraction nonconforming, geometric random variable, quality control, cumulative count of conforming chart, economic design.

\section{Introduction.}

This paper attempts to apply the concept of cumulative count of conforming chart (CCC chart) to monitor a system with three states.

Statistical process control is a widely used tool in the industry for process monitoring. Very commonly, control charts are merely used for the purpose of "fire fighting" for removal of assignable causes each time when the process parameter has shifted. However, occurrence of assignable causes can sometimes be the result of gradual deterioration of 
the system such as aging of parts. Thus, signals on the control charts may be used as indications of whether inspection or maintenance of the system should be carried out, rather than just the state of fitness of the process. Tagaras and Lee (1988) and Tagaras(1998) separated the $\bar{X}$-chart into several zones and optimized the chart for monitoring a process whose deterioration can be classified into two states in which one state requires minor restoration and the other requires major restoration, and considered economic design of the control chart. Cassady et al (2000) considered economic design of control charts for optimization of preventive maintenance policies for systems. See also Page (1955), Weindling et al (1970), Gordon and Weindling (1975), Montgomery (1980), Chan et al (2000), Montgomery (2001), Chan et al (2002), Chan (2003), Liao et al (2006) for related work.

In what follows, we shall assume that as production continues, the production system will deteriorate from its normal state to a state that requires minor maintenance, and if minor maintenance is not carried out the system will eventually deteriorate to a state that requires major maintenance. We shall use the CCC chart defined based on the geometric distribution to develop inspection and maintenance policies for a production system in which each item produced can be classified as either conforming or nonconforming. The CCC chart, first introduced by Calvin (1983), further developed by Goh (1987), and extended by and Ranjan et al (2003), is an alternative to the classical $n p$ - and $p$-charts that have serious drawbacks when the process nonconforming rate is low (Calvin(1983), Lucas(1989), Chan (2000), and Chan et al (1997)). The CCC chart can be used effectively for monitoring high-quality processes in which the fraction of nonconforming is low, even as low as at the level of ppm (parts per million) or ppb (parts per billion).

\section{The states and investigations of a system}


We consider process monitoring and maintenance of a system in which the fraction of nonconforming items produced is $p \in(0,1)$, and all items are inspected. The number of items inspected, $n$, until a nonconforming item is observed, is a geometric random variable with the following probability function $f(n)$ and cumulative distribution function $F(n)$ :

$$
f(n)=p(1-p)^{n-1}, \quad F(n)=1-(1-p)^{n} \quad(n=1,2, \ldots) .
$$

In monitoring such a system using the CCC chart, control limits are obtained from $F(n)$ at $p=p_{0}$, and an observed value of $n$ falling beyond the control limits is a signal for out of control which indicates possible shift of $p$ from its target value $p_{0}$.

Assume that the system has three states, the normal state $\mathrm{S}_{0}$ with nonconforming fraction $p=p_{0}>0$, the slightly deteriorated state $\mathrm{S}_{1}$ with $p=p_{1}>p_{0}$, and the seriously deteriorated state $\mathrm{S}_{2}$ with $p=p_{2}>p_{1}$. (Throughout this paper we shall use $\mathrm{S}_{0}, \mathrm{~S}_{1}$, $\mathrm{S}_{2}$ with Roman type "S" to denote the states of a system, while $S_{1}, S_{2}$ with italic " $S$ " will be reserved to represent mathematical quantities. Likewise, in what follows, $\mathrm{m}_{\gamma}$, $\mathrm{M}_{\gamma}, \mathrm{i}_{\gamma}, \mathrm{I}_{\gamma}$ and $\mathrm{s}_{\gamma}(\gamma=0,1,2$ or $1+2)$ with Roman type "m", "M", "i", "I" and "s" will be used to denote "maintenance", "inspection" and "signal" as appropriate, while $m, i, s$ in italic (with or without subscript) will be reserved to represent mathematical quantities.) Suppose that two grades of maintenance actions, minor maintenance $\mathrm{m}_{1}$ and major maintenance $\mathrm{m}_{2}$ are available, where $\mathrm{m}_{1}$ is less thorough than $\mathrm{m}_{2}$ but is less costly. For notational convenience, from time to time we shall use $\mathrm{m}_{0}$ to mean that no maintenance action will be carried out. Based on practical consideration, we assume the following:

1. No maintenance is required for $\mathrm{S}_{0}$, and when the system is in $\mathrm{S}_{0}$ neither $\mathrm{m}_{1}$ nor $\mathrm{m}_{2}$ will change its state.

2. When the system is in $\mathrm{S}_{0}$, it will deteriorate to $\mathrm{S}_{1}$ with probability $\pi_{01}>0$, imme- 
diately before an item is produced.

3. Either $m_{1}$ or $m_{2}$ can bring the system from $S_{1}$ back to $S_{0}$.

4. When the system is in $\mathrm{S}_{1}$, it will deteriorate to $\mathrm{S}_{2}$ with probability $\pi_{12}>0$, immediately before an item is produced.

5. $\mathrm{m}_{1}$ has no effect on $\mathrm{S}_{2}$, but $\mathrm{m}_{2}$ can bring the system from $\mathrm{S}_{2}$ to $\mathrm{S}_{0}$.

6. The system will not deteriorate from $\mathrm{S}_{0}$ to $\mathrm{S}_{2}$ directly without passing through $\mathrm{S}_{1}$.

7. Complete failure (that is, malfunctioning) of the system does not occur, even though as the system deteriorates from $\mathrm{S}_{0}$ to $\mathrm{S}_{1}$ and then to $\mathrm{S}_{2}$, the nonconforming fraction $p$ increases.

Suppose that $n_{1}>n_{2}>0$. Divide the set of all positive integers $Z^{+}$into the following three sets:

$$
\begin{aligned}
& Z_{0}=\left\{n \in Z^{+}: n_{1}+1 \leq n<\infty\right\} \\
& Z_{1}=\left\{n \in Z^{+}: n_{2}+1 \leq n \leq n_{1}\right\} \\
& Z_{2}=\left\{n \in Z^{+}: 1 \leq n \leq n_{2}\right\}
\end{aligned}
$$

If a nonconforming item is observed at $n \in Z_{\gamma}(\gamma=0,1,2)$, a type $\gamma$ signal $\mathrm{s}_{\gamma}$ is said to have occurred. When $\mathrm{s}_{0}$ has occurred, no inspection of the system will be carried out, and inspection will be carried out when $\mathrm{s}_{1}$ or $\mathrm{s}_{2}$ has occurred. When the system is in $\mathrm{S}_{0}$, we expect that $s_{0}$ will occur most of the time, and thus $s_{0}$ is regarded as an indication that the system is in its normal state $\mathrm{S}_{0}$. As the system deteriorates ( $p$ increases), the chance for $\mathrm{s}_{1}$ to occur increases. When the system is in $\mathrm{S}_{2}$, we expect that $\mathrm{s}_{2}$ will occur most of the time, and thus we regard $s_{2}$ as an indication that the system is in the most deteriorated state $\mathrm{S}_{2}$. However, any of $\mathrm{s}_{0}, \mathrm{~s}_{1}, \mathrm{~s}_{2}$ may occur when the system is in $\mathrm{S}_{0}, \mathrm{~S}_{1}$ or $\mathrm{S}_{2}$. Thus, when the system is in $\mathrm{S}_{0}$, signal $\mathrm{s}_{1}$ or $\mathrm{s}_{2}$ (which indicates system deterioration) may be regarded as "Type I error", and when the system is in $\mathrm{S}_{2}$, signal $\mathrm{s}_{0}$ or $\mathrm{s}_{0}$ (which 
indicates that the system is in state $\mathrm{S}_{0}$ or $\mathrm{S}_{1}$ ) may be regarded as "Type II error". In Section 6, we shall take into account such errors in economic design of inspection and maintenance policies where expressions for the expected numbers of $\mathbf{s}_{\gamma}(\gamma=0,1,2)$ when the system is $\mathrm{S}_{0}, \mathrm{~S}_{1}$ or $\mathrm{S}_{2}$ will be required, and such expressions will be given in Section 5 .

Suppose that two types of inspection, minor inspection $\mathrm{i}_{1}$ and major inspection $\mathrm{i}_{2}$, are available, where $\mathrm{i}_{1}$ is not as thorough as $\mathrm{i}_{2}$ but is less costly. For notational convenience, from time to time we shall use $\mathrm{i}_{0}$ to mean that no investigation will be carried out. When the system is in $\mathrm{S}_{0}$ or $\mathrm{S}_{1}$, both $\mathrm{i}_{1}$ and $\mathrm{i}_{2}$ will correctly reveal the true state of the system, but when the system is in $\mathrm{S}_{2}, \mathrm{i}_{1}$ will incorrectly indicate that the system is in $\mathrm{S}_{1}$ and only $\mathrm{i}_{2}$ will correctly reveal that the system is in $\mathrm{S}_{2}$.

From the economic of view, whether to carry out inspection depends on the inspection cost relative to the maintenance cost, and to the penalty of wrong indication of the state of the system. Consider the following three inspection arrangements, $\mathrm{I}_{1+2}, \mathrm{I}_{2}, \mathrm{I}_{0}$, and three maintenance arrangements $\mathrm{M}_{1+2}, \mathrm{M}_{2}, \mathrm{M}_{0}$.

$I_{1+2}-$ Both $i_{1}$ and $i_{2}$ are employed. When $s_{\gamma}$ occurs, $i_{\gamma}$ will be carried out $(\gamma=1,2)$. $\mathrm{I}_{2}$ - Only $\mathrm{i}_{2}$ is employed. When either $\mathrm{s}_{1}$ or $\mathrm{s}_{2}$ occurs, $\mathrm{i}_{2}$ will be carried out.

$\mathrm{I}_{0}-$ Neither $\mathrm{i}_{1}$ nor $\mathrm{i}_{2}$ will be employed. When $\mathrm{s}_{1}$ or $\mathrm{s}_{2}$ occurs, maintenance work will be carried out without first inspecting the system.

$\mathrm{M}_{1+2}-$ Both $\mathrm{m}_{1}$ and $\mathrm{m}_{2}$ are employed.

$\mathrm{M}_{2}$ - Only $\mathrm{m}_{2}$ is employed.

$\mathrm{M}_{0}-$ Neither $\mathrm{m}_{1}$ nor $\mathrm{m}_{2}$ is employed. That is, no maintenance work will be carried out.

The above concepts on states of the system and types of inspection and maintenance are illustrated by the following real-life example in maintenance of buses in a bus com- 
pany. A dynamo supplies the power to a cooling fan in the cooling system of the engine. By design, unfortunately this dynamo is installed near the engine with high temperature, and a cooling fan system called System C is designed for cooling the dynamo.

(a) $\mathrm{S}_{0}$ is the state where System C is not blocked by dust and operates efficiently, and the coolant will reach its boiling point with a small probability $p_{0}>0$ at the end of a day's operation.

(b) Checking whether the coolant has reached it boiling point at the end of a day's operation is "inspection of an item".

(c) $\mathrm{S}_{1}$ is the state where System $\mathrm{C}$ is partially block by dust, so that the dynamo is over-heated and supplies insufficient electric current to drive the cooling fan, and as a result the probability for the coolant to reach its boiling point at the end of a day's operation will be $p_{1}>p_{0}$.

(d) After a day's operation in state $S_{0}$, a bus will change to $S_{1}$ with probability $\pi_{01}>0$.

(e) Minor inspection $\mathrm{i}_{1}$ opens the chassis cover and inspects whether System C requires dust cleaning. Minor maintenance $\mathrm{m}_{1}$ removes the dust accumulated in System C. If $\mathrm{m}_{1}$ is not performed, System $\mathrm{C}$ will be eventually blocked by dust resulting in over-heating and dynamo damage, which is state $\mathrm{S}_{2}$.

(f) After a day's operation in state $\mathrm{S}_{1}$, a bus will change to $\mathrm{S}_{2}$ with probability $\pi_{12}>0$. In state $\mathrm{S}_{2}$, there is a probability $p_{2}\left(>p_{1}\right)$ for the coolant to reach its boiling point which could possibly result in engine damage.

(g) Major inspection $\mathrm{i}_{2}$ disassembles System $\mathrm{C}$ and inspects whether the dynamo has been damaged due to over-heating, and major maintenance $\mathrm{m}_{2}$ is to replace the dynamo.

(h) The values of $p_{0}, p_{1}, p_{2}, \pi_{01}$, and $\pi_{12}$ depend on the bus route.

(i) The costs for performing $\mathrm{i}_{1}, \mathrm{i}_{2}, \mathrm{~m}_{1}$, and $\mathrm{m}_{2}$ depend on the model of the bus. 


\section{Cycle of operation for the system.}

A cycle of operation is said to be completed when the system starts at $\mathrm{S}_{0}$, deteriorates to $\mathrm{S}_{1}$ or $\mathrm{S}_{2}$, and then is restored back to $\mathrm{S}_{0}$ by $\mathrm{m}_{1}$ or $\mathrm{m}_{2}$.

According to the framework of inspection and maintenance defined in Section 2, to study the system analytically we need to consider different scenarios for a cycle to complete as shown in Figure 1. The small circles, "o's", denote nonconforming items that either do not lead to any maintenance action or lead to a maintenance action that does not change the state of the system. The bullet, "•", denotes the last nonconforming item in a cycle that leads to maintenance action that restores the system from states $\mathrm{S}_{1}$ or $\mathrm{S}_{2}$ back to $\mathrm{S}_{0}$. Hence a cycle ends only after a bullet $\bullet$ has occured. Seven different cases (Case 0, ..., Case 6) are possible, and they are described below. A cycle may end under one of the six cases, Case $1, \ldots$, Case 6.
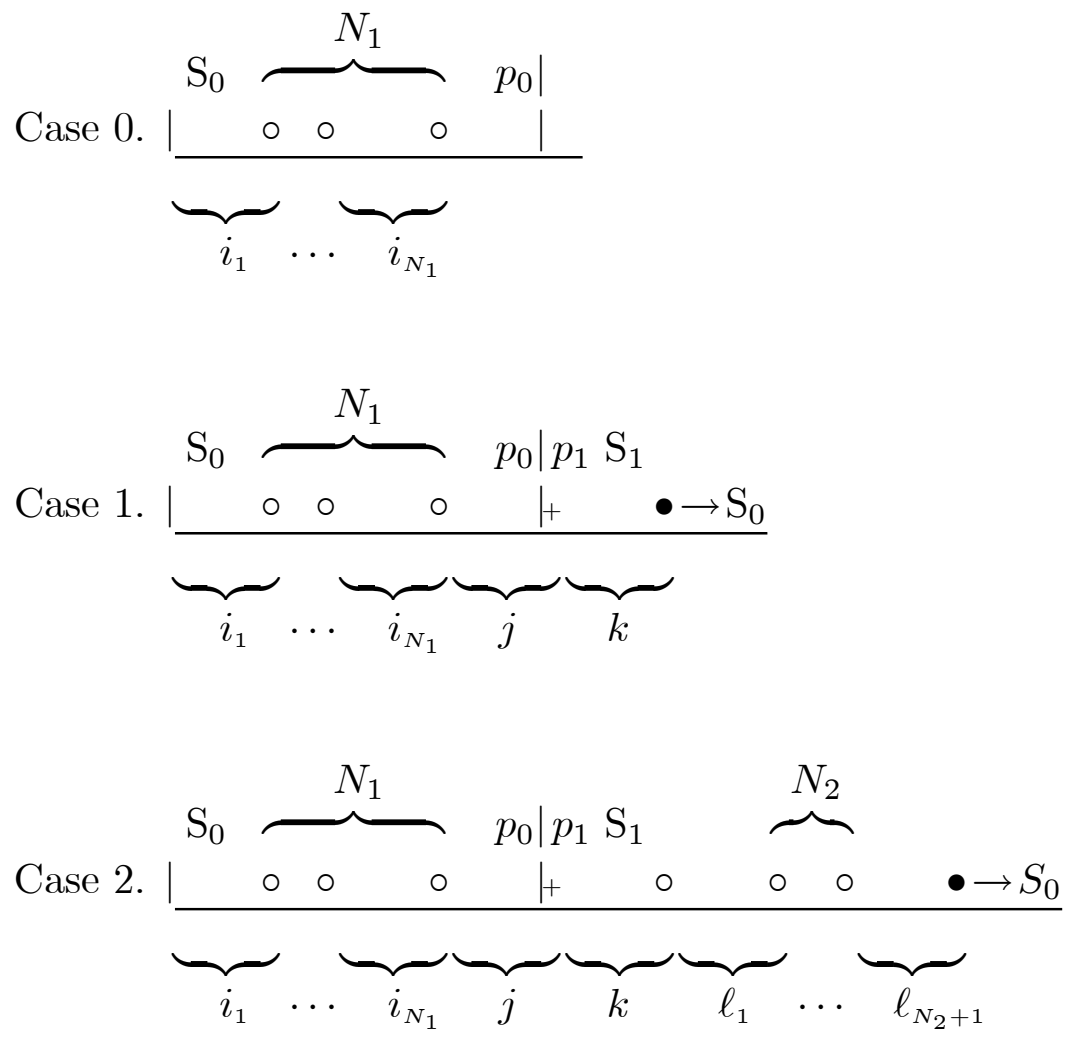

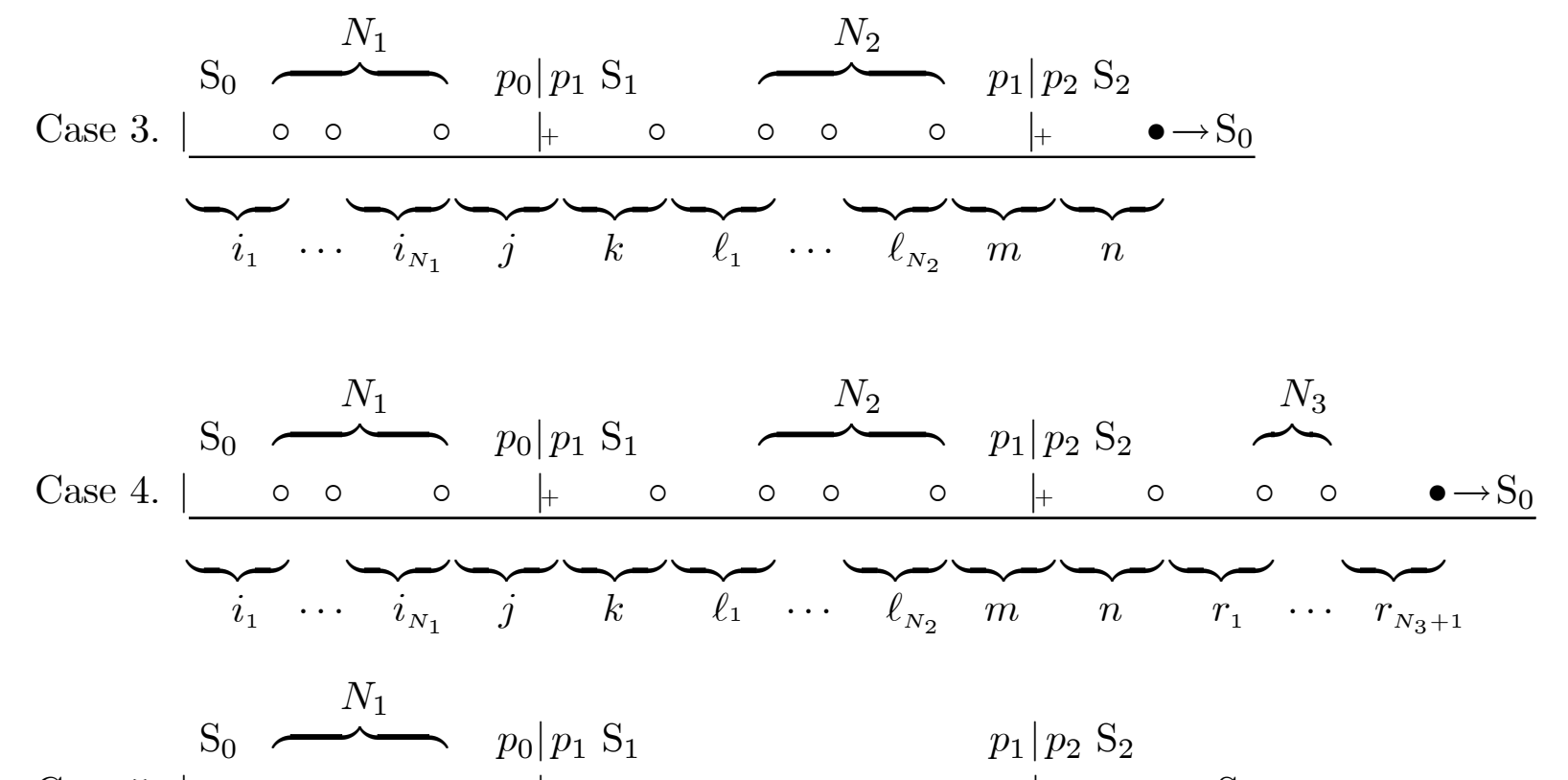

Case 5.
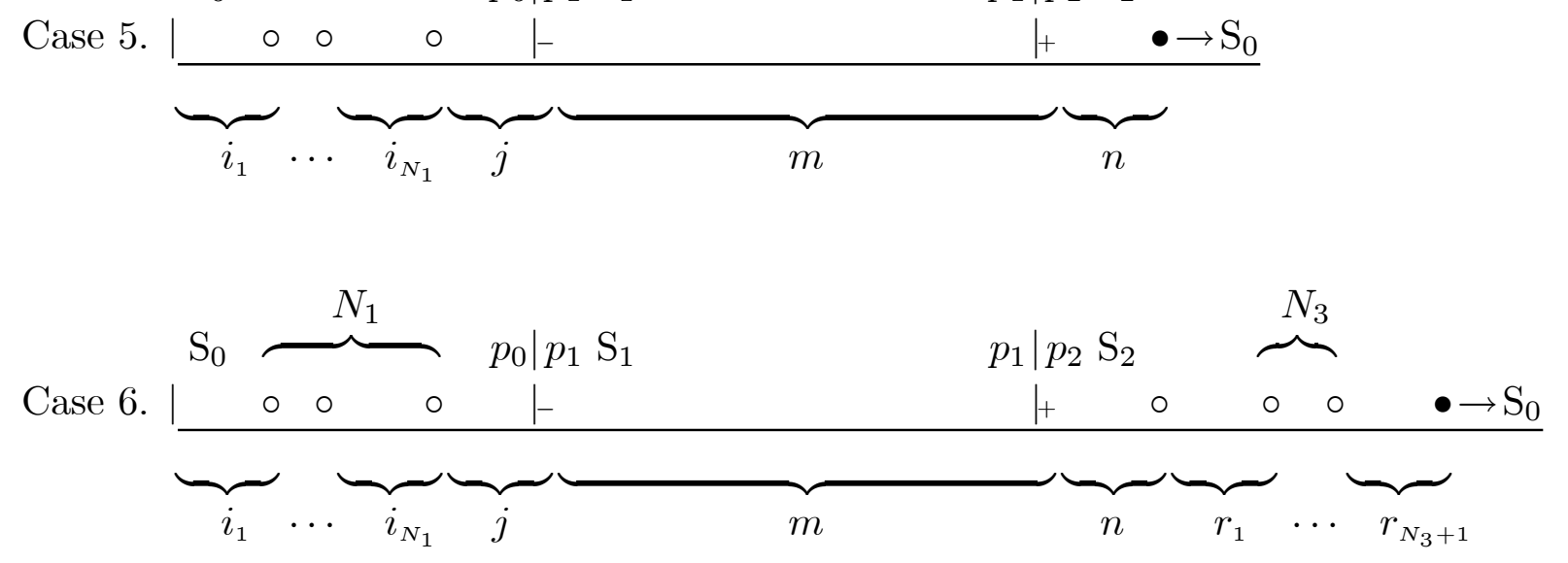

Figure 1. Occurrence of nonconformity items.

Case 0. While the system is in $\mathrm{S}_{0}, N_{1}(\geq 0)$ nonconforming items o's have occurred. As the system is in $\mathrm{S}_{0}$ and has not yet changed to $\mathrm{S}_{1}$, no maintenance action is necessary, there is no bullet $\bullet$ in the figure, and a cycle has not yet been formed. The figure shows that before a nonconforming item o occurs, $i_{u}-1\left(u=1, \ldots, N_{1}\right)$ conforming items are produced.

Case 1. While the system is in $\mathrm{S}_{0}, N_{1}(\geq 0)$ nonconforming items o's have occurred. Next, another $j-1$ conforming items have also occurred which is followed by changing 
of the system from $\mathrm{S}_{0}$ to $\mathrm{S}_{1}$, and then the $j^{\text {th }}$ item occurs. Immediately after the system has changed from $\mathrm{S}_{0}$ to $\mathrm{S}_{1}, k+1(\geq 1)$ items are produced, where the first $k$ of these $k+1$ items are conforming items but the last one is a $\bullet$. The plus sign "_" represents the first item produced immediately after the system has changed to $\mathrm{S}_{1}$, where this item is a conforming item if $k>0$, and is a nonconforming item $\bullet$ if $k=0$.

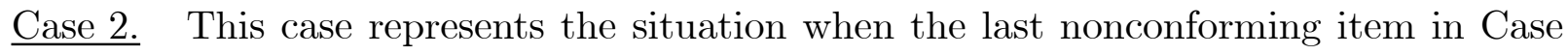
1 is not a $\bullet$ but a $\circ$. In this case, when the system is in $\mathrm{S}_{1}, N_{2}+1(\geq 1)$ o's occur before the $\bullet$. The plus sign " " represents a conforming item if $k>0$ and represents a nonconforming item $\circ$ if $k=0$. The figure shows that when the system is in $\mathrm{S}_{1}, \ell_{u}-1(\geq 0)$ $\left(u=1, \ldots, N_{2}+1\right)$ items are produced before each of the last $N_{2}+1$ nonconforming items.

Case 3. This case represents the situation when the last nonconforming item in Case 2 is not a $\bullet$ but a $\circ$. In this case, when the system is in $\mathrm{S}_{1}, N_{2}+1(\geq 1)$ o's have occurred. Next, another $m-1$ conforming items have occurred which is followed by changing of the system from $\mathrm{S}_{1}$ to $\mathrm{S}_{2}$, and then the $m^{\text {th }}$ item occurs. Immediately after the system has changed from $\mathrm{S}_{1}$ to $\mathrm{S}_{2}, n+1(\geq 1)$ items are produced, where the first $n$ of these $n+1$ items are conforming items but the last one is a $\bullet$. The plus sign "_" above the embrace of " $m$ " represents the first item produced immediately after the system has changed to $\mathrm{S}_{2}$, where this item is a conforming item if $n>0$ and is a nonconforming item $\bullet$ if $n=0$.

$\underline{\text { Case 4. }}$ This case represents the situation when the last nonconforming item in Case 3 is not a $\bullet$ but a $\circ$. In this case, immediately after the system has changed from $\mathrm{S}_{1}$ to $\mathrm{S}_{2}$, $N_{3}+1(\geq 1)$ o's have occurred before the bullet $\bullet$. The plus sign "+" above the embrace of " $m$ " represents a conforming item if $n>0$ and represents a nonconforming item $\circ$ if $n=0$. The figure shows that when the system is in $\mathrm{S}_{2}, r_{u}-1(\geq 0)\left(u=1, \ldots, N_{3}+1\right)$ 
conforming items are produced before each of the last $N_{3}+1$ nonconforming items.

In Cases 5 and 6 , no nonconforming item occurs when the system is in $\mathrm{S}_{1}$, and the bar "_" denotes a conforming item.

Case 5. While the system is in $\mathrm{S}_{0}, N_{1}(\geq 0)$ nonconforming items o's have occurred. Next, another $j-1$ conforming items have occurred which is followed by changing of the system from $\mathrm{S}_{0}$ to $\mathrm{S}_{1}$, and then the $j^{\text {th }}$ item occurs which is a conforming one. When the system is in $\mathrm{S}_{1}$, all the $m$ items produced are conforming. Immediately after the system has changed from $S_{1}$ to $S_{2}, n+1(\geq 1)$ items are produced, where the first $n$ of these $n+1$ items are conforming but the last one is a $\bullet$. The plus sign " " represents a conforming item if $n>0$, and represents the $\bullet$ if $n=0$.

Case 6. This the case represents the situation when the last nonconforming item in Case 5 is not a $\bullet$ but a $\circ$. In this case, immediately after the system has changed from $\mathrm{S}_{1}$ to $\mathrm{S}_{2}$, $N_{3}+1(\geq 1)$ o's have occurred before the bullet $\bullet$. The plus sign "+" above the embrace of " $m$ " represents a conforming item if $n>0$ and represents a nonconforming item $\circ$ if $n=0$. The figure shows that when the system is in $\mathrm{S}_{2}, r_{u}-1(\geq 0)\left(u=1, \ldots, N_{3}+1\right)$ conforming items are produced before each of the last $N_{3}+1$ nonconforming items.

Because a nonconforming item can only show up as a circle $\circ$ or a bullet $\bullet$ but not both, from the different positions of occurrences of the bullets •'s in Figure 1, it is not difficult to see that Case $1, \ldots$, Case 6 are mutually exclusive. These cases are also exhaustive for the system to complete a cycle, because a bullet $\bullet$ can occur only when the system is in either stage $\mathrm{S}_{1}$ or $S_{2}$, and Case $1, \ldots$, Case 6 include all possible cases for the bullet $\bullet$ to occur. At the end of Section 4 we shall prove that the total probability of occurrence of these six cases is 1 , which confirms that these cases are exhaustive. 


\section{Quantitative Analysis of the Model $\left(\mathbf{I}_{1+2}, \mathbf{M}_{1+2}\right)$}

In this section, we obtain the probabilities and expected items inspected for each of Case $0, \ldots$, Case 6 . Based on these results, expected numbers of inspections and maintenance actions can be derived, and using these results optimal inspection and maintenance strategies can be obtained in Section 7 .

Consider the inspection and maintenance model $\left(\mathrm{I}_{1+2}, \mathrm{M}_{1+2}\right)$, where all of $\mathrm{i}_{1}, \mathrm{i}_{2}, \mathrm{~m}_{1}$ and $\mathrm{m}_{2}$ are employed. Under this model, when $\mathrm{s}_{\gamma}(\gamma=1,2)$ appears, $\mathrm{i}_{\gamma}$ will be carried out. If an inspection shows that the system is in $\mathrm{S}_{\gamma}, \mathrm{m}_{\gamma}$ will be carried out $(\gamma=1,2)$. When the system is in $\mathrm{S}_{0}$, the o's in Figure 1 may indicate $\mathrm{s}_{0}, \mathrm{~s}_{1}$ or $\mathrm{s}_{2}$. When the system is in $\mathrm{S}_{1}$, the o's indicate $\mathrm{s}_{0}$, and the bullet $\bullet$ indicates either $\mathrm{s}_{1}$ or $\mathrm{s}_{2}$. When the system is in $\mathrm{S}_{2}$, the o's may indicate either $\mathrm{s}_{0}$ or $\mathrm{s}_{1}$, and the $\bullet$ indicates $\mathrm{s}_{2}$.

In what follows, for convenience we let $\beta_{0}=\left(1-\pi_{01}\right)\left(1-p_{0}\right), \beta_{1}=\left(1-\pi_{12}\right)\left(1-p_{1}\right)$.

In Case 0, while the system remains in $\mathrm{S}_{0}$, the probability for $(i-1)$ conforming items occurring followed by a nonconforming item $\circ$ is $\left(1-\pi_{01}\right)^{i}\left(1-p_{0}\right)^{i-1} p_{0}=\beta_{0}^{i-1}\left(1-\pi_{01}\right) p_{0}$. The total probability over the entire range of $i$ is

$$
\begin{aligned}
I_{.} & =I_{. ; i}=\sum_{i=1}^{\infty} \beta_{0}^{i-1}\left(1-\pi_{01}\right) p_{0}=\frac{\left(1-\pi_{01}\right) p_{0}}{1-\beta_{0}}=\left(\sum_{i \in Z_{0}}+\sum_{i \in Z_{1}}+\sum_{i \in Z_{2}}\right) \beta_{0}^{i-1}\left(1-\pi_{01}\right) p_{0} \\
& =I_{0 ; i}+I_{1 ; i}+I_{2 ; i}=I_{0}+I_{1}+I_{2},
\end{aligned}
$$

where $I_{0 ; i}, I_{1 ; i}, I_{2 ; i}$ denote the last three summations, respectively, and for simplicity, we also denote them by $I_{0}, I_{1}, I_{2}$, respectively. (In what follows, similar notations will be introduced to denote different summations.) The expected value of $i$ in Case 0 is

$$
\begin{aligned}
I .[i] & =I_{\cdot ; i}[i]=\sum_{i=1}^{\infty} i \beta_{0}^{i-1}\left(1-\pi_{01}\right) p_{0}=\frac{\left(1-\pi_{01}\right) p_{0}}{\left(1-\beta_{0}\right)^{2}}=\frac{I_{(0)}}{1-\beta_{0}} \\
& =\left(\sum_{i \in Z_{0}}+\sum_{i \in Z_{1}}+\sum_{i \in Z_{2}}\right) i \beta_{0}^{i-1}\left(1-\pi_{01}\right) p_{0}=I_{0 ; i}[i]+I_{1 ; i}[i]+I_{2 ; i}[i] .
\end{aligned}
$$


Refer to Case 1. Consider the following sequence of events: (A1) $j-1(\geq 0)$ consecutive conforming items are produced while the system remains in $\mathrm{S}_{0},(\mathrm{~A} 2)$ the system changes from $\mathrm{S}_{0}$ to $\mathrm{S}_{1}$, (A3) $k(\geq 0)$ consecutive conforming items are produced while the system remains in $\mathrm{S}_{1}$, and (A4) when the system is in $\mathrm{S}_{1}$, the $(k+1)^{t h}$ item produced is nonconforming. The probability for the events (A1) - (A4) to occur in sequence is $\left(1-\pi_{01}\right)^{j-1}\left(1-p_{0}\right)^{j-1} \pi_{01} \times\left(1-\pi_{12}\right)^{k}\left(1-p_{1}\right)^{k} p_{1}=\beta_{0}^{j-1} \beta_{1}^{k} \pi_{01} p_{1}$. The total probability over the entire ranges of $j$ and $k$ is

$$
\begin{aligned}
J K . & =J K_{\cdot ; j, k}=\sum_{j=1}^{\infty} \sum_{k=0}^{\infty} \beta_{0}^{j-1} \beta_{1}^{k} \pi_{01} p_{1}=\frac{\pi_{01}}{1-\beta_{0}} \times \frac{p_{1}}{1-\beta_{1}} \\
& =\left(\sum_{\substack{j+k \in Z_{0} \\
j \geq 1 ; k \geq 0}}+\sum_{\substack{j+k \in Z_{1} \\
j \geq 1 ; k \geq 0}}+\sum_{\substack{j+k \in Z_{2} \\
j \geq 1 ; k \geq 0}}\right) \beta_{0}^{j-1} \beta_{1}^{k} \pi_{01} p_{1} \\
& =J K_{0 ; j, k}+J K_{1 ; j, k}+J K_{2 ; j, k}=J K_{0}+J K_{1}+J K_{2} .
\end{aligned}
$$

The expected value of $j+k$ for Case 1 is

$$
\begin{aligned}
J K_{\cdot ; j, k}[j+k] & =J K_{j, k}[j+k]=\sum_{j=1}^{\infty} \sum_{k=0}^{\infty}(j+k) \beta_{0}^{j-1} \beta_{1}^{k} \pi_{01} p_{1} \\
& =\left(\sum_{\substack{j+k \in Z_{0} \\
j \geq 1 ; k \geq 0}}+\sum_{\substack{j+k \in Z_{1} \\
j \geq 1 ; k \geq 0}}+\sum_{\substack{j \geq k \in Z_{2} \\
j \geq 1 ; k \geq 0}}\right)(j+k) \beta_{0}^{j-1} \beta_{1}^{k} \pi_{01} p_{1} \\
& =J K_{0 ; j, k}[j+k]+J K_{1 ; j, k}[j+k]+J K_{2 ; j, k}[j+k] .
\end{aligned}
$$

In Case 2 , while the system is in $\mathrm{S}_{1}$, the probability for $(\ell-1)$ consecutive conforming item to occur followed by a nonconforming item $\circ$ is $\left(1-\pi_{12}\right)^{\ell}\left(1-p_{1}\right)^{\ell-1} p_{1}=\beta_{1}^{\ell-1}(1-$ $\left.\pi_{12}\right) p_{1}$. The total probability over the entire range of $\ell$ is

$$
\begin{aligned}
L .= & L_{. ; \ell}=\sum_{\ell=1}^{\infty} \beta_{1}^{\ell-1}\left(1-\pi_{12}\right) p_{1}=\frac{\left(1-\pi_{12}\right) p_{1}}{1-\beta_{1}}=\left(\sum_{\ell \in Z_{0}}+\sum_{\ell \in Z_{1}}+\sum_{\ell \in Z_{2}}\right) \beta_{1}^{\ell-1}\left(1-\pi_{12}\right) p_{1} \\
& =L_{0 ; \ell}+L_{1 ; \ell}+L_{2 ; \ell}=L_{0}+L_{1}+L_{2} .
\end{aligned}
$$


The expected value of $\ell$ in Case 2 is

$$
\begin{aligned}
& L_{* ; \ell}[\ell]=L_{\ell}[\ell]=\sum_{\ell=1}^{\infty} \ell \beta_{1}^{\ell-1}\left(1-\pi_{12}\right) p_{1}=\frac{\left(1-\pi_{12}\right) p_{1}}{\left(1-\beta_{1}\right)^{2}} \\
& =\left(\sum_{\ell \in Z_{0}}+\sum_{\ell \in Z_{1}}+\sum_{\ell \in Z_{2}}\right) \ell \beta_{1}^{\ell-1}\left(1-\pi_{12}\right) p_{1}=L_{0 ; \ell}[\ell]+L_{1 ; \ell}[\ell]+L_{2 ; \ell}[\ell] .
\end{aligned}
$$

Refer to Case 3. Consider the following sequence of events: (B1) $m-1(\geq 0)$ consecutive conforming items are produced while the system remains in $S_{1}$, (B2) the system changes from $\mathrm{S}_{1}$ to $\mathrm{S}_{2}$, (B3) $n(\geq 0)$ consecutive conforming items are produced while the system remains in $\mathrm{S}_{2}$, and (B4) when the system is in $\mathrm{S}_{2}$, the $(n+1)^{\text {th }}$ item produced is nonconforming. The probability for the events (B1) - (B4) to occur in sequence is $\left(1-\pi_{12}\right)^{m-1}\left(1-p_{1}\right)^{m-1} \pi_{12} \times\left(1-p_{2}\right)^{n} p_{2}=\beta_{1}^{m-1} \pi_{12}\left(1-p_{2}\right)^{n} p_{2}$. The total probability over the entire ranges of $m$ and $n$ is

$$
\begin{aligned}
M N . & =M N_{\cdot ; m, n}=\sum_{m=1}^{\infty} \sum_{n=0}^{\infty} \beta_{1}^{m-1} \pi_{12}\left(1-p_{2}\right)^{n} p_{2}=\frac{\pi_{12}}{1-\beta_{1}} \\
& =\left(\sum_{\substack{m+n \in Z_{0} \\
m \geq 1 ; n \geq 0}}+\sum_{\substack{m+n \in Z_{1} \\
m \geq 1 ; n \geq 0}}+\sum_{\substack{m+n \in Z_{2} \\
m \geq 1 ; n \geq 0}}\right) \beta_{1}^{m-1} \pi_{12}\left(1-p_{2}\right)^{n} p_{2} \\
& =M N_{0 ; m, n}+M N_{1 ; m, n}+M N_{2 ; m, n}=M N_{0}+M N_{1}+M N_{2} .
\end{aligned}
$$

The corresponding expected number of items inspected for Case 3 is

$$
\begin{aligned}
& M N .[m+n]=M N_{\cdot ; m, n}[m+n]=\sum_{m=1}^{\infty} \sum_{n=0}^{\infty}(m+n) \beta_{1}^{m-1} \pi_{12}\left(1-p_{2}\right)^{n} p_{2} \\
= & \sum_{m=1}^{\infty} \sum_{n=0}^{\infty} m \beta_{1}^{m-1} \pi_{12}\left(1-p_{2}\right)^{n} p_{2}+\sum_{m=1}^{\infty} \sum_{n=0}^{\infty} n \beta_{1}^{m-1} \pi_{12}\left(1-p_{2}\right)^{n} p_{2} \\
= & \frac{\pi_{12}}{\left(1-\beta_{1}\right)^{2}} \times+\frac{\pi_{12}}{1-\beta_{1}} \times \frac{1}{p_{2}}=\frac{\pi_{12}\left(p_{2}+1-\beta_{1}\right)}{\left(1-\beta_{1}\right)^{2} p_{2}} \\
= & \left(\sum_{\substack{m+n \in Z_{0} \\
m \geq 1 ; n \geq 0}}+\sum_{\substack{m+n \in Z_{1} \\
m \geq 1 ; n \geq 0}}+\sum_{\substack{m+n \in Z_{2} \\
m \geq 1 ; n \geq 0}}\right)(m+n) \beta_{1}^{m-1} \pi_{12}\left(1-p_{2}\right)^{n} p_{2} \\
= & M N_{0 ; m, n}[m+n]+M N_{1 ; m, n}[m+n]+M N_{2 ; m, n}[m+n] .
\end{aligned}
$$


In Case 4 , when the system is in $\mathrm{S}_{2}$, the probability for $(r-1)$ conforming to be produced followed by a nonconforming item is $\left(1-p_{2}\right)^{r-1} p_{2}$. The total probability over the entire range of $r$ is

$$
\begin{aligned}
R . & =R_{; ; r}=\sum_{r=1}^{\infty}\left(1-p_{2}\right)^{r-1} p_{2}=1=\left(\sum_{r \in Z_{0}}+\sum_{r \in Z_{1}}+\sum_{r \in Z_{2}}\right)\left(1-p_{2}\right)^{r-1} p_{2} \\
& =R_{0 ; r}+R_{1 ; r}+R_{2 ; r}=R_{0}+R_{1}+R_{2} .
\end{aligned}
$$

The expected value of $r$ for Case 4 is

$$
\begin{aligned}
R .[r] & =R_{\bullet ; r}[r]=\sum_{r=1}^{\infty} r\left(1-p_{2}\right)^{r-1} p_{2}=\frac{1}{p_{2}}=\left(\sum_{r \in Z_{0}}+\sum_{r \in Z_{1}}+\sum_{r \in Z_{2}}\right) r\left(1-p_{2}\right)^{r-1} p_{2} \\
& =R_{0 ; r}[r]+R_{1 ; r}[r]+R_{2 ; r}[r] .
\end{aligned}
$$

Refer to Case 5. Consider the events: (C1) immediately after the system has changes from $\mathrm{S}_{0}$ to $\mathrm{S}_{1}, m(\geq 1)$ consecutive conforming items are produced, (C2) the system changes from $\mathrm{S}_{1}$ to $\mathrm{S}_{2}$, (C3) immediately after the system has changes from $\mathrm{S}_{1}$ to $\mathrm{S}_{2}$, $n(\geq 0)$ consecutive conforming items are produced while the systems remains in $\mathrm{S}_{2}$, (C4) when the systems is in $\mathrm{S}_{2}$, the $(n+1)^{t h}$ item produced is nonconforming. The probability for events (A1), (A2), (C1), (C2), (C3), (C4) to occur in sequence is $\left(1-\pi_{01}\right)^{j-1}(1-$ $\left.\left.p_{0}\right)^{j-1} \pi_{01}\left(1-p_{1}\right) \times\left(1-\pi_{12}\right)^{m-1}\left(1-p_{1}\right)^{m-1} \pi_{12} \times\left(1-p_{2}\right)^{n} p_{2}\right]=\beta_{0}^{j-1} \beta_{1}^{m-1} \pi_{01}(1-$ $\left.p_{1}\right) \pi_{12}\left(1-p_{2}\right)^{n} p_{2}$. The total probability over the entire ranges of $j, m, n$, is

$$
\begin{aligned}
J M N . & =J M N_{\cdot j, m, n}=\sum_{j=1}^{\infty} \sum_{m=1}^{\infty} \sum_{n=0}^{\infty} \beta_{0}^{j-1} \beta_{1}^{m-1} \pi_{01}\left(1-p_{1}\right) \pi_{12}\left(1-p_{2}\right)^{n} p_{2} \\
& =\frac{\pi_{01}}{1-\beta_{0}} \times \frac{\pi_{12}\left(1-p_{1}\right)}{1-\beta_{1}} \\
& =\left(\begin{array}{c}
\left.\sum_{\substack{j+m+n \in Z_{0} \\
j \geq 1 ; m \geq 1 ; n \geq 0}}+\sum_{\substack{j \geq m+n \in Z_{1} \\
j \geq 1 ; m \geq 1 ; n \geq 0}}^{\substack{j \geq 1 ; m \geq 1 ; n \geq 0 \\
j+m \in Z_{2}}}\right) \\
=
\end{array} \beta_{0}^{j-1} \beta_{1}^{m-1} \pi_{01}\left(1-p_{1}\right) \pi_{12}\left(1-p_{2}\right)^{n} p_{2}\right. \\
& J M N_{0 ; j, m, n}+J M N_{1 ; j, m, n}+J M N_{2 ; j, m, n}=J M N_{0}+J M N_{1}+J M N_{2} .
\end{aligned}
$$


The expected value of $j+m+n$ for Case 5 is

$$
\begin{aligned}
& J M N .=J M N_{\cdot j, m, n}[j+m+n] \\
& =\sum_{j=1}^{\infty} \sum_{m=1}^{\infty} \sum_{n=0}^{\infty}(j+m+n) \beta_{0}^{j-1} \beta_{1}^{m-1} \pi_{01}\left(1-p_{1}\right) \pi_{12}\left(1-p_{2}\right)^{n} p_{2} \\
& =\frac{\pi_{01}}{\left(1-\beta_{0}\right)^{2}} \times \frac{\pi_{12}\left(1-p_{1}\right)}{1-\beta_{1}}+\frac{\pi_{01}}{1-\beta_{0}} \times \frac{\pi_{12}\left(1-p_{1}\right)}{\left(1-\beta_{1}\right)^{2}}+\frac{\pi_{01}}{1-\beta_{0}} \times \frac{\pi_{12}\left(1-p_{1}\right)}{1-\beta_{1}} \times \frac{1}{p_{2}} \\
& =\frac{\pi_{01} \pi_{12}\left(1-p_{1}\right)}{\left(1-\beta_{0}\right)^{2}\left(1-\beta_{1}\right)^{2} p_{2}}\left(\frac{1}{1-\beta_{0}}+\frac{1}{1-\beta_{1}}+\frac{1}{p_{2}}\right) \\
& =\left(\sum_{\substack{j+m+n \in Z_{0} \\
j \geq 1 ; m \geq 1 ; n \geq 0}}+\sum_{\substack{j+m+n \in Z_{1} \\
j \geq 1 ; m \geq 1 ; n \geq 0}}+\sum_{\substack{j+m+n \in Z_{2} \\
j \geq 1 ; m \geq 1 ; n \geq 0}}(j+m+n) \beta_{0}^{j-1} \beta_{1}^{m-1} \pi_{01}\left(1-p_{1}\right) \pi_{12}\left(1-p_{2}\right)^{n} p_{2}\right. \\
& =J M N_{0 ; j, m, n}[j+m+n]+J M N_{1 ; j, m, n}[j+m+n]+J M N_{2 ; j, m, n}[j+m+n] .
\end{aligned}
$$

In Case 6, when the system is in $\mathrm{S}_{2}$, the probability for $(r-1)$ conforming to be produced followed by a nonconforming item is $\left(1-p_{2}\right)^{r-1} p_{2}$. The total probability over the entire range of $r$ and the expected value of $r$ are the same as those in Case 4, that is,

$$
\begin{aligned}
R . & =R_{\cdot ; r}=\sum_{r=1}^{\infty}\left(1-p_{2}\right)^{r-1} p_{2}=1=\left(\sum_{r \in Z_{0}}+\sum_{r \in Z_{1}}+\sum_{r \in Z_{2}}\right)\left(1-p_{2}\right)^{r-1} p_{2} \\
& =R_{0 ; r}+R_{1 ; r}+R_{2 ; r}=R_{0}+R_{1}+R_{2}, \\
R .[r] & =R_{\cdot ; r}[r]=\sum_{r=1}^{\infty} r\left(1-p_{2}\right)^{r-1} p_{2}=\frac{1}{p_{2}}=\left(\sum_{r \in Z_{0}}+\sum_{r \in Z_{1}}+\sum_{r \in Z_{2}}\right) r\left(1-p_{2}\right)^{r-1} p_{2} \\
& =R_{0 ; r}[r]+R_{1 ; r}[r]+R_{2 ; r}[r] .
\end{aligned}
$$


Let $P_{1}, \ldots, P_{6}$ be the probabilities for Cases $1, \ldots, 6$ to occur, respectively. We have

$$
\begin{aligned}
P_{1}= & \sum_{N_{1}=0}^{\infty} I_{\cdot}^{N_{1}}\left(J K_{1}+J K_{2}\right) \\
P_{2}= & \sum_{N_{1}=0}^{\infty} I_{\cdot}^{N_{1}} J K_{0} \times \sum_{N_{2}=0}^{\infty} L_{0}^{N_{2}}\left(L_{1}+L_{2}\right) \\
P_{3}= & \sum_{N_{1}=0}^{\infty} I_{\cdot}^{N_{1}} J K_{0} \times \sum_{N_{2}=0}^{\infty} L_{0}^{N_{2}} M N_{2} \\
P_{4}= & \sum_{N_{1}=0}^{\infty} I^{N_{1}} J K_{0} \times \sum_{N_{2}=0}^{\infty} L_{0}^{N_{2}}\left(M N_{0}+M N_{1}\right) \times \sum_{N_{3}=0}^{\infty}\left(R_{0}+R_{1}\right)^{N_{3}} R_{2} \\
= & \sum_{N_{1}=0}^{\infty} I^{N_{1}} J K_{0} \times \sum_{N_{2}=0}^{\infty} L_{0}^{N_{2}}\left(M N_{0}+M N_{1}\right), \\
P_{5}= & \sum_{N_{1}=0}^{\infty} I_{\cdot}^{N_{1}} J M N_{2}, \\
P_{6}= & \sum_{N_{1}=0}^{\infty} I_{\cdot}^{N_{1}}\left(J M N_{0}+J M N_{1}\right) \times \sum_{N_{3}=0}^{\infty}\left(R_{0}+R_{1}\right)^{N_{3}} R_{2}=\sum_{N_{1}=0}^{\infty} I_{\cdot}^{N_{1}}\left(J M N_{0}+J M N_{1}\right) .
\end{aligned}
$$

Since Case $1, \ldots$, Case 6 are mutually exclusive, the sum $\left(P_{1}+P_{2}+\left(P_{3}+P_{4}\right)\right)+$ $\left(P_{5}+P_{6}\right)=1$ ensures that these six cases include all the possible cases for the system to complete a cycle.

\section{Expected values associated with models $\left(\mathbf{I}_{1+2}, \mathbf{M}_{1+2}\right)$ and $\left(\mathbf{I}_{0}, \mathbf{M}_{1+2}\right)$}

We first consider the model $\left(\mathrm{I}_{1+2}, \mathrm{M}_{1+2}\right)$. In what follows, for any function $g(\nu)$ the summation $\sum_{\nu=u}^{w} g(\nu)$ will be interpreted as 0 if $u>w$.

The closed form of the formulas displayed below are established using the formulas for the summations $S_{1}, S_{11}, S_{2}, S_{22}, S_{3}, S_{33}$ given in the Appendix.

From the definitions of $I_{.}, I_{0}, I_{1}, I_{2}, J_{0}, J_{1}, \ldots, J M N_{2}$, we shall derive the expressions for the expected number of items inspected $E_{\eta}[\mathrm{isp}]$, the expected number of type $\gamma$ $(\gamma=0,1,2)$ signals $E_{\eta}\left[\mathrm{s}_{\gamma}\right]$, the expected number of type $\gamma$ investigation $E_{\eta}\left[\mathrm{i}_{\gamma}\right]$, and the expected number type $\gamma$ maintenance $E_{\eta}\left[\mathrm{m}_{\gamma}\right]$ for Case $1, \ldots$, Case 6 . 
For convenience in presentation, we shall use the notation

$$
\begin{aligned}
& I .=I_{. ; i}=\sum_{i=1}^{\infty} \beta_{0}^{i-1}\left(1-\pi_{01}\right) p_{0}, \quad I_{\cdot ; \text { isp }}=I_{\cdot ; i}[i]=\sum_{i=1}^{\infty} \beta_{0}^{i-1}\left(1-\pi_{01}\right) p_{0} \times i, \ldots, \\
& J K_{1+2 ; \text { isp }}=J K_{1 ; \text { isp }}+J K_{2 ; \text { isp }}=J K_{1+2 ; j, k}[j+k]=\sum_{j+k \in Z_{1} \cup Z_{2}} \beta_{0}^{j-1} \beta_{1}^{k} \pi_{01} p_{1} \times(j+k), \ldots,
\end{aligned}
$$

and so on.

In all the cases in the Figure, when the system is in $\mathrm{S}_{0}$, each of the $N_{1}$ circles o's represents a nonconforming item which gives either a type 0 signal $\mathrm{s}_{0}$, a type 1 signal $\mathrm{s}_{1}$, or a type 2 signal $\mathrm{s}_{2}$; since these three types of signal occur in the ratio $I_{0}: I_{1}: I_{2}$ and $I .=I_{0}+I_{1}+I_{2}$, the expected numbers of type 0 , type 1 and type 2 signals are $\frac{I_{0}}{I} \times N_{1}, \frac{I_{1}}{I} \times N_{1}$ and $\frac{I_{2}}{I} \times N_{1}$, respectively. When the system is in $\mathrm{S}_{1}$, a circle $\circ$ represents a nonconforming item which gives a signal $\mathrm{s}_{0}$. When the system is in $\mathrm{S}_{2}$, a circle $\circ$ represents a nonconforming item which gives either a signal $\mathrm{s}_{0}$ or $\mathrm{s}_{1}$.

When the system is in $\mathrm{S}_{1}$, a bullet $\bullet$ represents a nonconforming item which gives either a signal $\mathrm{s}_{1}$ or $\mathrm{s}_{2}$. When the system is in $\mathrm{S}_{2}$, a bullet $\bullet$ represents a nonconforming item which gives a signal $\mathrm{s}_{2}$.

Since $\mathrm{s}_{\gamma}$ causes $\mathrm{i}_{\gamma}(\gamma=0,1,2)$, for all $\eta=1, \ldots, 6$ we have

$$
E_{1}\left[\mathrm{i}_{0}\right]=E_{1}\left[\mathrm{~s}_{0}\right], \quad E_{1}\left[\mathrm{i}_{1}\right]=E_{1}\left[\mathrm{~s}_{1}\right], \quad E_{1}\left[\mathrm{i}_{2}\right]=E_{1}\left[\mathrm{~s}_{2}\right]
$$

Derivations of the expected numbers of inspections and signals are based on the expressions of the probabilities $P_{1}, \ldots, P_{6}$ given in Section 4 . The calculations are straightforward but cumbersome, and here an illustration is given for Case 1. Corresponding formulas for Cases $2-6$ can be obtained in a similar way, but the expressions are lengthy and they will not be displayed here.

Expected numbers associated with Case 1. 
For Case 1, it is shown from Figure 1 that $N_{1}$ circles o's have occurred, and that inspection is required if and only if $j+k \in Z_{1}$ or $j+k \in Z_{2}$. The expected number of items inspected is $E_{1}[\mathrm{isp}]=\underset{N_{1}}{E}\left[\underset{i_{1}}{E} \cdots\left[\underset{i_{N_{1}}}{E}\left[\underset{j, k}{E}\left[i_{1}+\cdots+i_{N_{1}}+j+k\right]\right]\right]\right]$. Here when $N_{1}=0$, we interpret the sum $i_{1}+\cdots+i_{N_{1}}$ as 0 and interpret $I_{\cdot ; i_{1}} \cdots I_{\cdot ; i_{N_{1}}}$ in the following derivation for $E_{1}[$ isp] as 1. (This convention will be used for the rest of this paper.)

We recall that the probability associated with Case 1 is

$$
\begin{aligned}
P_{1} & =\sum_{N_{1}=0}^{\infty}\left\{\sum_{i_{1}=1}^{\infty} \beta_{0}^{i_{1}-1}\left(1-\pi_{01}\right) p_{0} \cdots \sum_{i_{N_{1}}=1}^{\infty} \beta_{0}^{i_{N_{1}}-1}\left(1-\pi_{01}\right) p_{0}\right\} \times \sum_{\substack{j+k \in Z_{1} \cup Z_{2} \\
j \geq 1 ; k \geq 0}} \beta_{0}^{j-1} \pi_{01} p_{1} \\
& =\sum_{N_{1}=0}^{\infty}\left\{I_{\cdot ; i_{1}} \cdots I_{\cdot ; i_{N_{1}}}\right\} J K_{1+2 ; j, k}=\sum_{N_{1}=0}^{\infty} I_{\cdot}^{N_{1}}\left(J K_{1}+J K_{2}\right),
\end{aligned}
$$

It follows that

$$
\begin{aligned}
& E_{1}[\mathrm{isp}]=\underset{N_{1}}{E}\left[\underset{i_{1}}{E} \cdots\left[i_{i_{N_{1}}}^{E}\left[\underset{j, k}{E}\left[i_{1}+\cdots+i_{N_{1}}+j+k\right]\right]\right]\right] \\
& =\sum_{N_{1}=0}^{\infty}\left\{\sum_{i_{1}=1}^{\infty} \beta_{0}^{i_{1}-1}\left(1-\pi_{01}\right) p_{0} \cdots \sum_{i_{N_{1}}=1}^{\infty} \beta_{0}^{i_{N_{1}}-1}\left(1-\pi_{01}\right) p_{0}\right\} \times \\
& \sum_{\substack{j+k \in Z_{1} \cup Z_{2} \\
j \geq 1 ; k \geq 0}} \beta_{0}^{j-1} \pi_{01} p_{1}\left[i_{1}+\cdots+i_{N_{1}}+j+k\right] \\
& =\sum_{N_{1}=0}^{\infty}\left\{I_{\cdot ; i_{1}} \cdots I_{\cdot ; i_{N_{1}}}\right\} J K_{1+2 ; j, k}\left[i_{1}+\cdots+i_{N_{1}}+j+k\right] \\
& =\sum_{N_{1}=0}^{\infty}\left(I_{\cdot ; i_{1}}\left[i_{1}\right] \times I_{\cdot ; i_{2}} \times \cdots \times I_{\cdot ; i_{N_{1}}} \times J K_{1+2 ; j, k}+\right. \\
& \text { : } \\
& I_{\cdot ; i_{1}} \times \cdots \times I_{\cdot ; i_{N_{1}-1}} \times I_{\cdot ; i_{N_{1}}}\left[i_{N_{1}}\right] \times J K_{1+2 ; j, k}+ \\
& \left.I_{: ; i_{1}} \times I_{\cdot ; i_{N_{1}}} \times \cdots \times I_{: ; i_{N_{1}}} \times J K_{1,2 ; j, k}[j+k]\right) \\
& =\sum_{N_{1}=0}^{\infty}\left(N_{1} I_{\cdot ; \text {;sp }} I^{N_{1}-1}\left(J K_{1}+J K_{2}\right)+I_{\cdot}^{N_{1}} J K_{1+2 ; \text { isp }}\right) \\
& =\frac{I_{. ; \mathrm{isp}}\left(J K_{1}+J K_{2}\right)}{(1-I .)^{2}}+\frac{J K_{1+2 ; \mathrm{isp}}}{1-I .} .
\end{aligned}
$$


For Case 1, as shown in Figure 1 the last of the $j+k$ items inspected indicated by the bullet $\bullet$ gives either a signal $\mathrm{s}_{1}$ or $\mathrm{s}_{2}$, and the expected number of occurrence of these two types of signal are in the ratio $J K_{1}: J K_{2}$. Hence from the expression for $P_{1}$ we have

$$
\begin{aligned}
E_{1}\left[\mathrm{~s}_{0}\right] & =\sum_{N_{1}=0}^{\infty} I^{N_{1}}\left(J K_{1}+J K_{2}\right)\left(\frac{I_{0}}{I .} \times N_{1}\right)=\frac{I_{0}\left(J K_{1}+J K_{2}\right)}{(1-I .)^{2}}, \\
E_{2}\left[\mathrm{~s}_{1}\right] & =\sum_{N_{1}=0}^{\infty} I_{\cdot}^{N_{1}}\left(J K_{1}+J K_{2}\right)\left(\frac{I_{1}}{I .} \times N_{1}+\frac{J K_{1}}{J K_{1}+J K_{2}} \times 1\right) \\
& =\frac{I_{1}\left(J K_{1}+J K_{2}\right)}{(1-I .)^{2}}+\frac{J K_{1}}{1-I .}, \\
E_{2}\left[\mathrm{~s}_{2}\right] & =\sum_{N_{1}=0}^{\infty} I_{\cdot}^{N_{1}}\left(J K_{1}+J K_{2}\right)\left(\frac{I_{2}}{I .} \times N_{1}+\frac{J K_{2}}{J K_{1}+J K_{2}} \times 1\right) \\
& =\frac{I_{2}\left(J K_{1}+J K_{2}\right)}{(1-I .)^{2}}+\frac{J K_{2}}{1-I .} .
\end{aligned}
$$

When the system is in $\mathrm{S}_{0}$, no maintenance will be carried out. When the system is in $S_{1}$, either $i_{1}$ or $i_{2}$ will indicate the true state of the system, and $m_{1}$ will be carried out, while $\mathrm{m}_{2}$ will not be necessary. Thus, looking at Case 1 in Figure 1 and using the expressions of $E_{1}\left[\mathrm{~s}_{1}\right]$ and $E_{1}\left[\mathrm{~s}_{2}\right]$, we obtain

$$
\begin{aligned}
& E_{1}\left[\mathrm{~m}_{1}\right]=\sum_{N_{1}=0}^{\infty} I^{N_{1}}\left(J K_{1}+J K_{2}\right)\left(\frac{J K_{1}+J K_{2}}{J K_{1}+J K_{2}} \times 1\right)=P_{1}, \\
& E_{1}\left[\mathrm{~m}_{2}\right]=0 .
\end{aligned}
$$

Model $\left(\mathrm{I}_{0}, \mathrm{M}_{1+2}\right)$.

As for this model investigations will not be carried out, and signal $\mathrm{s}_{\gamma}$ will immediately trigger maintenance action $\mathrm{m}_{\gamma}(\gamma=0,1,2)$. This situation occurs when the costs of investigations $i_{1}$ and $i_{2}$ are nearly as high as the costs of maintenance $m_{1}$ and $m_{2}$, so that it is economically not viable to carry out investigation. Under the model $\left(\mathrm{I}_{0}, \mathrm{M}_{1+2}\right)$, all the expressions for $P_{1}, \ldots, P_{6}$ are identical to those of the model $\left(\mathrm{I}_{1+2}, \mathrm{M}_{1+2}\right)$, and so 
are the expressions $E_{1}\left[\right.$ isp] $, \ldots, E_{6}[$ isp] for expected number of items inspected, and the expressions $E_{1}\left[\mathrm{~s}_{i}\right], \ldots, E_{6}\left[\mathrm{~s}_{i}\right]$ for the expected numbers of type $i$ signals $(i=0,1,2)$. It is clear that

$$
E_{\eta}\left[\mathrm{i}_{\gamma}\right]=0, E_{\eta}\left[\mathrm{m}_{\gamma}\right]=E_{\eta}\left[\mathrm{s}_{\gamma}\right] \quad(\eta=1, \ldots, 6 ; \gamma=0,1,2)
$$

\section{Other models without minor investigation.}

When the cost of minor investigation $i_{1}$ is nearly as high as $i_{2}$, it will be economically viable to replace $i_{1}$ by $i_{2}$. When the costs of $i_{1}$ and $i_{2}$ are nearly as high as $m_{1}$ and $m_{2}$, it will be economically viable to replace $i_{1}$ by $m_{1}$ and replace $i_{2}$ by $m_{2}$. When the cost of $\mathrm{m}_{1}$ is nearly as high as $\mathrm{m}_{2}$, it will be economically viable to replace $\mathrm{m}_{1}$ by $\mathrm{m}_{2}$.

Under the models $\left(\mathrm{I}_{2}, \mathrm{M}_{1+2}\right),\left(\mathrm{I}_{2}, \mathrm{M}_{2}\right)$ and $\left(\mathrm{I}_{0}, \mathrm{M}_{2}\right)$, when the system is in $\mathrm{S}_{0}$, the o's in Figure 1 may indicate signals $\mathrm{s}_{0}, \mathrm{~s}_{1}$ or $\mathrm{s}_{2}$. When the system is in $\mathrm{S}_{1}$, the o's indicate signal $\mathrm{s}_{0}$, and the bullet $\bullet$ indicate either $\mathrm{s}_{1}$ or $\mathrm{s}_{2}$. When the system is in $\mathrm{S}_{2}$, the o's indicate $\mathrm{s}_{0}$, and the $\bullet$ indicate either $\mathrm{s}_{1}$ or $\mathrm{s}_{2}$. Under these models, either $\mathrm{s}_{1}$ or $\mathrm{s}_{2}$ will cause $\mathrm{i}_{2}$ or $\mathrm{i}_{0}$, and therefore $n_{2}$ is irrelevant and only $n_{1}$ will appear in the formulas for various probabilities and expected values. (For the definitions of $n_{1}, n_{2}$, refer to the sets $Z_{0}, Z_{1}, Z_{2}$ defined in Section 1.) Under the model $\left(\mathrm{I}_{0}, \mathrm{M}_{0}\right)$, neither investigation nor maintenance will be carried out, and both $n_{1}$ and $n_{2}$ are irrelevant.

\subsection{Model $\left(\mathbf{I}_{2}, \mathbf{M}_{1+2}\right)$.}

Under this model, when either $\mathrm{s}_{1}$ or $\mathrm{s}_{2}$ appears, $\mathrm{i}_{2}$ will be carried out. If $\mathrm{i}_{2}$ shows that the system is in state $\mathrm{S}_{\gamma}, \mathrm{m}_{\gamma}(\gamma=1,2)$ will be carried out which will restore the system back to $\mathrm{S}_{0}$.

The probabilities for Cases 1 and 2 in Figure 1 to occur are identical to those shown in Section 4 for models $\left(\mathrm{I}_{1+2}, \mathrm{M}_{1+2}\right)$ and $\left(\mathrm{I}_{0}, \mathrm{M}_{1+2}\right)$; and thus the expected values $E_{\eta}$ [isp], 
$E_{\eta}\left[\mathrm{s}_{\gamma}\right]$ and $E_{\eta}\left[\mathrm{m}_{\gamma}\right](\eta=1,2, \gamma=0,1,2)$ are identical to those of the models $\left(\mathrm{I}_{1+2}, \mathrm{M}_{1+2}\right)$ and $\left(\mathrm{I}_{0}, \mathrm{M}_{1+2}\right)$. We denote the probabilities for Cases 3, 4, 5 and 6 in Figure 1 by $P_{3}^{*}, P_{4}^{*}, P_{5}^{*}$ and $P_{6}^{*}$, respectively, These probabilities are as follows.

$$
\begin{aligned}
P_{1} & =\sum_{N_{1}=0}^{\infty} I^{N_{1}}\left(J K_{1}+J K_{2}\right), \\
P_{2} & =\sum_{N_{1}=0}^{\infty} I^{N_{1}} J K_{0} \times \sum_{N_{2}=0}^{\infty} L_{0}^{N_{2}}\left(L_{1}+L_{2}\right) \\
P_{3}^{*} & =\sum_{N_{1}=0}^{\infty} I^{N_{1}} J K_{0} \times \sum_{N_{2}=0}^{\infty} L_{0}^{N_{2}}\left(M N_{1}+M N_{2}\right) \\
P_{4}^{*} & =\sum_{N_{1}=0}^{\infty} I^{N_{1}} J K_{0} \times \sum_{N_{2}=0}^{\infty} L_{0}^{N_{2}} M N_{0} \times \sum_{N_{3}=0}^{\infty} R_{0}^{N_{3}}\left(R_{1}+R_{2}\right) \\
& =\sum_{N_{1}=0}^{\infty} I^{N_{1}} J K_{0} \times \sum_{N_{2}=0}^{\infty} L_{0}^{N_{2}} M N_{0}, \\
P_{5}^{*} & =\sum_{N_{1}=0}^{\infty} I^{N_{1}}\left(J M N_{1}+J M N_{2}\right), \\
P_{6}^{*} & =\sum_{N_{1}=0}^{\infty} I_{\cdot}^{N_{1}} J M N_{0} \times \sum_{N_{3}=0}^{\infty} R_{0}^{N_{3}}\left(R_{1}+R_{2}\right)=\sum_{N_{1}=0}^{\infty} I_{\cdot}^{N_{1}} J M N_{0} .
\end{aligned}
$$

It is straightforward to show that $P_{3}^{*}+P_{4}^{*}=P_{3}+P_{4}$, and $P_{5}^{*}+P_{6}^{*}=P_{5}+P_{6}$.

Refer to Figure 1 for Cases 3, 4, 5, 6. Under the model $\left(\mathrm{I}_{2}, \mathrm{M}_{1+2}\right)$, when the system is in $\mathrm{S}_{1}$, the $1+N_{2}$ circles o's represent type 0 signals so's. In Cases 6 , when the system is in $\mathrm{S}_{2}$, the $1+N_{3}$ circles o's represent type 0 signals $\mathrm{s}_{0}$ 's, and the bullet $\bullet$ represents either a type 1 signal $\mathrm{s}_{1}$ or a type 2 signal $\mathrm{s}_{2}$, where these two types of signal occur in the ratio $R_{1}: R_{2}$.

Replacing $\mathrm{i}_{1}$ in the model $\left(\mathrm{I}_{1+2}, \mathrm{M}_{1+2}\right)$ by $\mathrm{i}_{2}$ forms the model $\left(\mathrm{I}_{2}, \mathrm{M}_{1+2}\right)$, and hence for model $\left(\mathrm{I}_{2}, \mathrm{M}_{1+2}\right)$ we have

$$
E_{\eta}\left[\mathrm{i}_{0}\right]=E_{\eta}\left[\mathrm{s}_{0}\right], \quad E_{\eta}\left[\mathrm{i}_{1}\right]=0, \quad E_{\eta}\left[\mathrm{i}_{2}\right]=E_{\eta}\left[\mathrm{s}_{1}\right]+E_{\eta}\left[\mathrm{s}_{2}\right] \quad(\eta=1, \ldots, 6) .
$$

For Cases 1 and 2, all the expected values of the number of signals, inspections and maintenance actions are identical to those for the model $\left(\mathrm{I}_{1+2}, \mathrm{M}_{1+2}\right)$. For Cases $3-6$, 
corresponding results can be obtained based on the probabilities $P_{3}^{*}, P_{4}^{*}, P_{5}^{*}, P_{6}^{*}$, and the detailed are omitted here.

\subsection{Model $\left(\mathbf{I}_{2}, \mathbf{M}_{2}\right)$.}

Under this model, an $\mathrm{s}_{1}$ or $\mathrm{s}_{2}$ will be followed by $\mathrm{i}_{2}$ which will correctly reveal the state of the system, and if the system is in $\mathrm{S}_{1}$ or $\mathrm{S}_{2}, \mathrm{~m}_{2}$ will be carried out which will restore the system back to $\mathrm{S}_{0}$. The expressions $P_{3}^{*}, \ldots, P_{6}^{*}$ for the probabilities of Cases $3, \ldots, 6$ in Figure 1 are as the same as those for the model $\left(\mathrm{I}_{2}, \mathrm{M}_{1+2}\right)$ in Sub-section 6.1. The expressions $P_{1}$ and $P_{2}$ for the probabilities of Cases 1 and 2 in Figure 1 are identical to those of the model $\left(\mathrm{I}_{1+2}, \mathrm{M}_{1+2}\right)$ in Section 4.

Since $i_{1}$ is not carried out, we have

$$
E_{\eta}\left[\mathrm{i}_{0}\right]=E_{\eta}\left[\mathrm{s}_{0}\right], \quad E_{\eta}\left[\mathrm{i}_{1}\right]=0, \quad E_{\eta}\left[\mathrm{i}_{2}\right]=E_{\eta}\left[\mathrm{s}_{1}\right]+E_{\eta}\left[\mathrm{s}_{2}\right] \quad(\eta=1, \ldots, 6) .
$$

For Cases 1 and 2 , it can be seen that $E_{1}\left[\mathrm{~m}_{0}\right]$ and $E_{2}\left[\mathrm{~m}_{0}\right]$ are also identical to those of the model $\left(\mathrm{I}_{1+2}, \mathrm{M}_{1+2}\right)$, and we have $E_{1}\left[\mathrm{~m}_{2}\right]=P_{1}, E_{2}\left[\mathrm{~m}_{2}\right]=P_{2}$.

Refer to Cases 3, 4, 5, 6 in the Figure 1. Under model $\left(\mathrm{I}_{2}, \mathrm{M}_{2}\right)$, when the system is in $\mathrm{S}_{1}$, the $1+N_{2}$ circles o's represent type 0 signals so's. In Case 4 , when the system is in $\mathrm{S}_{2}$, the $1+N_{3}$ circles o's represent type 0 signals $\mathrm{s}_{0}$ 's, and the bullet $\bullet$ represents either a type 0 signal $\mathrm{s}_{0}$ or a type 1 signal $\mathrm{s}_{1}$, where these two types of signal occur in the ratio $R_{1}: R_{2}$. Hence from the expression for the numbers of items inspected $E_{4}[\mathrm{isp}], E_{4}\left[\mathrm{~s}_{\gamma}\right], E_{4}[\mathrm{isp}], E_{4}\left[\mathrm{~s}_{\gamma}\right](\gamma=0,1,2)$ are identical to those of the model $\left(\mathrm{I}_{2}, \mathrm{M}_{1+2}\right)$.

In Cases 3, 4, 5 and 6, since $\mathrm{m}_{1}$ is absent and the $\bullet$ represent $\mathrm{m}_{2}$, the expressions for $E_{\eta}\left[\mathrm{m}_{\gamma}\right](\eta=3,4,5,6, \gamma=0,1,2)$ are identical to those of model $\left(\mathrm{I}_{2}, \mathrm{M}_{1+2}\right)$.

\subsection{Model $\left(\mathbf{I}_{0}, \mathbf{M}_{2}\right)$.}


Under this model, no inspection will be carried out, and $s_{1}$ or $s_{2}$ will always lead to $\mathrm{m}_{2}$. If the system is in $\mathrm{S}_{i}(i=1,2), \mathrm{m}_{2}$ will always restore the system back to $\mathrm{S}_{0}$. The expressions of the probabilities for Cases 1 and 2 in Figure 1 are the same as the $P_{1}$ and $P_{2}$ for model $\left(\mathrm{I}_{1+2}, \mathrm{M}_{1+2}\right)$ in Section 4 . The expressions for the probabilities for Case $3, \ldots, 6$ in Figure 1 and the expected number of items inspected are the same as the $P_{3}^{*}, \ldots, P_{6}^{*}$ and $E_{3}[\mathrm{isp}], \ldots, E_{6}[\mathrm{isp}]$ for model $\left(\mathrm{I}_{2}, \mathrm{M}_{1+2}\right)$ in Sub-Section 6.1.

For the model $\left(\mathrm{I}_{0}, \mathrm{M}_{2}\right)$, for all $\eta=1, \ldots, 6$ we have

$$
\begin{aligned}
& E_{\eta}\left[\mathrm{i}_{1}\right]=E_{\eta}\left[\mathrm{i}_{2}\right]=E_{\eta}\left[\mathrm{i}_{3}\right]=0, \\
& E_{\eta}\left[\mathrm{m}_{0}\right]=E_{\eta}\left[\mathrm{s}_{0}\right], E_{\eta}\left[\mathrm{m}_{1}\right]=0, E_{\eta}\left[\mathrm{m}_{2}\right]=E_{\eta}\left[\mathrm{s}_{1}\right]+E_{\eta}\left[\mathrm{s}_{2}\right] .
\end{aligned}
$$

\subsection{Model $\left(\mathbf{I}_{0}, \mathbf{M}_{0}\right)$.}

Under this model, none of $\mathrm{i}_{1}, \mathrm{i}_{2}, \mathrm{~m}_{1}$ and $\mathrm{m}_{1}$ will be employed. The system starts at $\mathrm{S}_{0}$, deteriorates to $\mathrm{S}_{1}$ if $\pi_{01}>0$, and then deteriorates to $\mathrm{S}_{2}$ if $\pi_{12}>0$. This model may be applied when the cost required to carry out investigation and maintenance are high compared to the penalty cost due to occurrence of nonconforming items.

\section{Economic design of inspection and maintenance policy}

In reality, various factors determine whether to perform investigation and maintenance on the system. These factors include resources available, operational convenience, loss due to system failure and nonconforming items, and others. Generally speaking, investigation and maintenance should be carried out if the loss due to occurrence of nonconforming items is considerable.

We consider the following three types of cost: (1) penalty $\operatorname{cost} C_{n c}$ due to occurrence of nonconforming items, (2) investigation cost $C_{i v s}$, (3) maintenance cost $C_{m}$. If $N_{t o l}$ is 
the expected total number of items produced in a cycle, the average total cost per item per cycle is

$$
\bar{c}=\left(C_{n c}+C_{i v s}+C_{m}\right) / N_{t o l} .
$$

In accordance with the investigation and maintenance policies defined in the previous sections, we consider the following fixed costs:

$$
\begin{aligned}
& c_{n c}=\text { the penalty cost incurred when a nonconforming item is produced, } \\
& c_{i v s, 1}=\text { cost of carrying out } \mathrm{i}_{1} \text { each time, } \\
& c_{i v s, 2}=\text { cost of carrying out } \mathrm{i}_{2} \text { each time, } \\
& c_{m, 1}=\text { cost of carrying out } \mathrm{m}_{1} \text { each time, } \\
& c_{m, 2}=\text { cost of carrying out } \mathrm{m}_{2} \text { each time. }
\end{aligned}
$$

In what follows, $\sum_{\eta}$ will denote the summation over $\eta=1, \ldots, 6$ for Cases $1, \ldots, 6$ in Figure 1. Since all items are inspected, for all the models $\left(\mathrm{I}_{1+2}, \mathrm{M}_{1+2}\right),\left(\mathrm{I}_{2}, \mathrm{M}_{1+2}\right)$, $\left(\mathrm{I}_{0}, \mathrm{M}_{1+2}\right),\left(\mathrm{I}_{2}, \mathrm{M}_{2}\right)$ and $\left(\mathrm{I}_{0}, \mathrm{M}_{2}\right)$ we have

$$
N_{t o l}=\sum_{\eta} E_{\eta}[\text { isp }]
$$

where the expressions for $E_{\eta}[$ isp] for the different models are given in Sections 4, 5, 6 (and so are the expressions for $E_{\eta}\left[\mathrm{s}_{0}\right], E_{\eta}\left[\mathrm{s}_{1}\right], E_{\eta}\left[\mathrm{s}_{2}\right], E_{\eta}\left[\mathrm{m}_{1}\right], E_{\eta}\left[\mathrm{m}_{2}\right]$ in the following $\left.C_{n c}, C_{i v s}, C_{m}\right)$.

Since each nonconforming item gives a signal (either $\mathrm{s}_{0}, \mathrm{~s}_{1}$ or $\mathrm{s}_{2}$ ), the expected total number of nonconforming items is $\sum_{\eta}\left(E_{\eta}\left[\mathrm{s}_{0}\right]+E_{\eta}\left[\mathrm{s}_{1}\right]+E_{\eta}\left[\mathrm{s}_{2}\right]\right)$, giving

$$
C_{n c}=c_{n c} \sum_{\eta}\left(E_{\eta}\left[\mathrm{s}_{0}\right]+E_{\eta}\left[\mathrm{s}_{1}\right]+E_{\eta}\left[\mathrm{s}_{2}\right]\right) .
$$

As for $C_{i v s}$ and $C_{m}$, we have

$$
\begin{aligned}
& C_{i v s}=c_{i v s, 1} \sum_{\eta} E_{\eta}\left[\mathrm{i}_{1}\right]+c_{i v s, 2} \sum_{\eta} E_{\eta}\left[\mathrm{i}_{2}\right], \\
& C_{m}=c_{m, 1} \sum_{\eta} E_{\eta}\left[\mathrm{m}_{1}\right]+c_{m, 2} \sum_{\eta} E_{\eta}\left[\mathrm{m}_{2}\right] .
\end{aligned}
$$


For the model $\left(\mathrm{I}_{0}, \mathrm{M}_{0}\right)$, the system will change to state $\mathrm{S}_{2}$ eventually and remain in $\mathrm{S}_{2}$ indefinitely. The average number of items produced when the system is in states $\mathrm{S}_{0}$ and $\mathrm{S}_{1}$ are $1 / \pi_{01}$ and $1 / \pi_{12}$, respectively. Therefore the average total cost is

$$
\bar{c}=\lim _{N \rightarrow \infty} \frac{p_{0} c_{n c} / \pi_{01}+p_{1} c_{n c} / \pi_{12}+p_{2} N c_{n c}}{1 / \pi_{01} E_{1}+1 / \pi_{12}+N}=p_{2} c_{n c}
$$

where $N$ denotes the number of items produced after the process has shifted to state $\mathrm{S}_{2}$.

Referring to the sets $Z_{0}, Z_{1}, Z_{2}$ defined in Section 2, we see that the $\bar{c}$ for each inspection and maintenance model can be minimized with respect to $n_{1}$ and $n_{2}$ in the region $\mathcal{N}=\left\{\left(n_{1}, n_{2}\right): 2 \leq n_{2}+1 \leq n_{1} \leq \infty\right\}$. Since $\bar{c}$ is an algebraic function of $n_{1}$ and $n_{2}$, minimization can be carried out easily using software such as Mathematica. The minimum of $\bar{c}$ for the six maintenance models, say $\bar{c}_{m i n}$, can be compared, and the most cost-effective model can be selected. It is clear that $\bar{c}$ depends on the parameters $p_{0}, p_{1}$, $p_{2}, \pi_{01}, \pi_{12}, c_{n c}, c_{i n v, 1}, c_{i n v, 2}, c_{m, 1}, c_{m, 2}$, and the minimum cost $\bar{c}_{m i n}$ may be attained under any of the five models $\left(\mathrm{I}_{1+2}, \mathrm{M}_{1+2}\right),\left(\mathrm{I}_{2}, \mathrm{M}_{1+2}\right),\left(\mathrm{I}_{0}, \mathrm{M}_{1+2}\right),\left(\mathrm{I}_{0}, \mathrm{M}_{2}\right),\left(\mathrm{I}_{0}, \mathrm{M}_{0}\right)$. Table 1 gives the numerical values of $\bar{c}$ for different values of the parameters and for different models. In each of the Cases $(\mathrm{a})-(\mathrm{h})$ in Table 1 , the minimum $\bar{c}$ 's among the different models are high-lighted with square brackets. In Cases (a), (b), (c), the minimum $\bar{c}$ is attained under model $\left(\mathrm{I}_{1+2}, \mathrm{M}_{1+2}\right)$. In Cases $(\mathrm{d}),(\mathrm{e}),(\mathrm{f}),(\mathrm{g})$, the minimum $\bar{c}$ is attained under the models $\left(\mathrm{I}_{0}, \mathrm{M}_{1+2}\right),\left(\mathrm{I}_{2}, \mathrm{M}_{1+2}\right),\left(\mathrm{I}_{0}, \mathrm{M}_{2}\right),\left(\mathrm{I}_{0}, \mathrm{M}_{0}\right)$, respectively.

Since $c_{m, 1}<c_{m, 2}$, given any values of $p_{0}, p_{1}, p_{2}, \pi_{01}, \pi_{12}, c_{n c}, c_{i n v, 1}, c_{i n v, 2}, c_{m, 1}$, $c_{m, 2}$, it is easy to see from the expressions of $C_{m}$ that the $\bar{c}$ for model $\left(\mathrm{I}_{2}, \mathrm{M}_{2}\right)$ cannot be smaller that of model $\left(\mathrm{I}_{2}, \mathrm{M}_{1+2}\right)$. This is based on the assumption that $c_{m, 2}$ is the same for both $\mathrm{M}_{2}$ and $\mathrm{M}_{1+2}$. However, in some cases an additional cost $\Delta$ per maintenance action is required to provide two types of maintenance services $m_{1}$ and $m_{2}$, rather than just a single type $m_{2}$. If $\Delta$ is included into the the maintenance cost, the cost to carry 
out $\mathrm{m}_{2}$ each time under the model $\left(\mathrm{I}_{2}, \mathrm{M}_{1+2}\right)$ will be $c_{m, 2}+\Delta$ which is larger than the cost $c_{m, 2}$ under the model $\left(\mathrm{I}_{2}, \mathrm{M}_{2}\right)$. In this case, it is possible that $\bar{c}_{\text {min }}$ under the model $\left(\mathrm{I}_{2}, \mathrm{M}_{2}\right)$ will be smaller than that under the model $\left(\mathrm{I}_{2}, \mathrm{M}_{1+2}\right)$, and this is illustrated by Case (h) in Table 1. This idea can be extended to the case when an additional cost is incurred in order to maintain two types of inspection (minor $i_{1}$ and major $i_{2}$ ), instead of just one type (major $\mathrm{i}_{2}$ ).

For models $\left(\mathrm{I}_{1+2}, \mathrm{M}_{1+2}\right)$ and $\left(\mathrm{I}_{0}, \mathrm{M}_{1+2}\right)$, the minimum of $\bar{c}$ can be attained in the interior or on the boundary of $\mathcal{N}$. Cases (a) and (g) in Table 1 illustrate that for model $\left(\mathrm{I}_{1+2}, \mathrm{M}_{1+2}\right)$, the minimum of $\bar{c}$ is attained in the interior of $\mathcal{N}$ (where $2<n_{2}+1<n_{1}<$ $\infty)$, and Cases (b), (c), (d), (e), (f), (h) illustrate that the minimum of $\bar{c}$ is attained on the boundary of $\mathcal{N}$ (where either $1<n_{2}<n_{1}=\infty, n_{2}=n_{1}=\infty$ or $2<n_{2}+1=n_{1}<\infty$ ). In Case (b), $n_{1}=\infty$ means that $\mathrm{i}_{0}$ does not exist, and occurrence of a nonconforming item is either $i_{1}$ or $i_{2}$. In Case (c), $n_{1}=n_{2}=\infty$ indicates that both $i_{0}$ and $i_{1}$ do not exist, and any occurrence of nonconforming item is $\mathrm{i}_{2}$.

Table 1. Numerical examples.

Here $\left(p_{0}, p_{1}, p_{2}, \pi_{01}, \pi_{12}\right)=(0.015,0.019,0.05,0.0004,0.0035)$.

(a) $\left(c_{n c}, c_{i n v, 1}, c_{i n v, 2}, c_{m, 1}, c_{m, 2}\right)=(2.3,4.8,10,4.9,260)$.

\begin{tabular}{|r|cc|lcc|c|}
\hline Investigation $=$ & $I_{1+2}$ & $\mathrm{I}_{0}$ & $\mathrm{I}_{2}$ & $\mathrm{I}_{2}$ & $\mathrm{I}_{0}$ & $\mathrm{I}_{0}$ \\
Maintenance $=$ & $\mathrm{M}_{1+2}$ & $\mathrm{M}_{1+2}$ & $\mathrm{M}_{1+2}$ & $\mathrm{M}_{2}$ & $\mathrm{M}_{2}$ & $\mathrm{M}_{0}$ \\
\hline $\bar{c}_{\min }=$ & {$[0.11024]$} & 0.16171 & 0.11365 & 0.13279 & 0.17553 & 0.115 \\
$N_{t o l}=$ & 2671.82 & 2834.03 & 2705.25 & 3141.35 & 3141.35 & $\infty$ \\
$n_{2}=$ & 6 & 1 & - & - & - & - \\
$n_{1}=$ & 17 & 15 & 9 & 1 & 1 & - \\
\hline
\end{tabular}

(b) $\left(c_{n c}, c_{i n v, 1}, c_{i n v, 2}, c_{m, 1}, c_{m, 2}\right)=(120,3,18,11,22)$. 


\begin{tabular}{|r|cc|lcc|c|}
\hline Investigation $=$ & $\mathrm{I}_{1+2}$ & $\mathrm{I}_{0}$ & $\mathrm{I}_{2}$ & $\mathrm{I}_{2}$ & $\mathrm{I}_{0}$ & $\mathrm{I}_{0}$ \\
Maintenance $=$ & $\mathrm{M}_{1+2}$ & $\mathrm{M}_{1+2}$ & $\mathrm{M}_{1+2}$ & $\mathrm{M}_{2}$ & $\mathrm{M}_{2}$ & $\mathrm{M}_{0}$ \\
\hline $\bar{c}_{\text {min }}=$ & {$[1.90466]$} & 1.93549 & 1.92969 & 1.93181 & 1.93550 & 6 \\
$N_{\text {tol }}=$ & 2559.19 & 2674.08 & 2666.93 & 2666.93 & 2679.38 & $\infty$ \\
$n_{2}=$ & 6 & 12 & - & - & - & - \\
$n_{1}=$ & $\infty$ & 13 & 14 & 14 & 12 & - \\
\hline
\end{tabular}

(c) $\left(c_{n c}, c_{i n v, 1}, c_{i n v, 2}, c_{m, 1}, c_{m, 2}\right)=(220,0.1,0.5,10,20)$.

\begin{tabular}{|r|cc|lcc|c|}
\hline Investigation $=$ & $\mathrm{I}_{1+2}$ & $\mathrm{I}_{0}$ & $\mathrm{I}_{2}$ & $\mathrm{I}_{2}$ & $\mathrm{I}_{0}$ & $\mathrm{I}_{0}$ \\
Maintenance $=$ & $\mathrm{M}_{1+2}$ & $\mathrm{M}_{1+2}$ & $\mathrm{M}_{1+2}$ & $\mathrm{M}_{2}$ & $\mathrm{M}_{2}$ & $\mathrm{M}_{0}$ \\
\hline $\bar{c}_{\text {min }}=$ & {$[3.33543]$} & 3.47997 & {$[3.33543]$} & 3.33876 & 3.48091 & 11 \\
$N_{\text {tol }}=$ & 2547.64 & 2633.66 & 2547.64 & 2547.64 & 2644.92 & $\infty$ \\
$n_{2}=$ & $\infty$ & 16 & - & - & - & - \\
$n_{1}=$ & $\infty$ & 24 & $\infty$ & $\infty$ & 19 & - \\
\hline
\end{tabular}

(d) $\left(c_{n c}, c_{i n v, 1}, c_{i n v, 2}, c_{m, 1}, c_{m, 2}\right)=(11,2.7,15,2.8,17)$.

\begin{tabular}{|r|cc|lcc|c|}
\hline Investigation $=$ & $\mathrm{I}_{1+2}$ & $\mathrm{I}_{0}$ & $\mathrm{I}_{2}$ & $\mathrm{I}_{2}$ & $\mathrm{I}_{0}$ & $\mathrm{I}_{0}$ \\
Maintenance $=$ & $\mathrm{M}_{1+2}$ & $\mathrm{M}_{1+2}$ & $\mathrm{M}_{1+2}$ & $\mathrm{M}_{2}$ & $\mathrm{M}_{2}$ & $\mathrm{M}_{0}$ \\
\hline $\bar{c}_{\text {min }}=$ & 0.20333 & {$[0.19967]$} & 0.20272 & 0.20490 & 0.20036 & 0.55 \\
$N_{\text {tol }}=$ & 2784.10 & 2754.99 & 2804.82 & 2804.82 & 2804.82 & $\infty$ \\
$n_{2}=$ & 4 & 3 & 1 & 1 & 1 & - \\
$n_{1}=$ & 5 & 9 & 4 & 4 & 4 & - \\
\hline
\end{tabular}

(e) $\left(c_{n c}, c_{i n v, 1}, c_{i n v, 2}, c_{m, 1}, c_{m, 2}\right)=(120,5,10,411,650)$.

\begin{tabular}{|r|cc|lcc|c|}
\hline Investigation $=$ & $\mathrm{I}_{1+2}$ & $\mathrm{I}_{0}$ & $\mathrm{I}_{2}$ & $\mathrm{I}_{2}$ & $\mathrm{I}_{0}$ & $\mathrm{I}_{0}$ \\
Maintenance $=$ & $\mathrm{M}_{1+2}$ & $\mathrm{M}_{1+2}$ & $\mathrm{M}_{1+2}$ & $\mathrm{M}_{2}$ & $\mathrm{M}_{2}$ & $\mathrm{M}_{0}$ \\
\hline $\bar{c}_{\text {min }}=$ & 2.11196 & 2.73581 & {$[2.08753]$} & 2.14071 & 2.58369 & 6 \\
$N_{\text {tol }}=$ & 2621.72 & 2824.78 & 2627.87 & 2648.57 & 2927.14 & $\infty$ \\
$n_{2}=$ & 27 & 3 & - & - & - & - \\
$n_{1}=$ & 28 & 4 & 25 & 18 & 2 & - \\
\hline
\end{tabular}

(f) $\left(c_{n c}, c_{i n v, 1}, c_{i n v, 2}, c_{m, 1}, c_{m, 2}\right)=(20,9,15,10,17)$.

\begin{tabular}{|r|cc|lcc|c|}
\hline Investigation $=$ & $\mathrm{I}_{1+2}$ & $\mathrm{I}_{0}$ & $\mathrm{I}_{2}$ & $\mathrm{I}_{2}$ & $\mathrm{I}_{0}$ & $\mathrm{I}_{0}$ \\
Maintenance $=$ & $\mathrm{M}_{1+2}$ & $\mathrm{M}_{1+2}$ & $\mathrm{M}_{1+2}$ & $\mathrm{M}_{2}$ & $\mathrm{M}_{2}$ & $\mathrm{M}_{0}$ \\
\hline $\bar{c}_{\min }=$ & 0.35242 & 0.34863 & 0.34984 & 0.35073 & {$[0.34742]$} & 1 \\
$N_{\text {tol }}=$ & 2736.77 & 2756.84 & 2748.31 & 2748.31 & 2770.65 & $\infty$ \\
$n_{2}=$ & 6 & 5 & - & - & - & - \\
$n_{1}=$ & 7 & 6 & 6 & 6 & 5 & - \\
\hline
\end{tabular}

(g) $\left(c_{n c}, c_{i n v, 1}, c_{i n v, 2}, c_{m, 1}, c_{m, 2}\right)=(1,4.8,10,4.9,260)$. 


\begin{tabular}{|r|cc|lcc|c|}
\hline Investigation $=$ & $\mathrm{I}_{1+2}$ & $\mathrm{I}_{0}$ & $\mathrm{I}_{2}$ & $\mathrm{I}_{2}$ & $\mathrm{I}_{0}$ & $\mathrm{I}_{0}$ \\
Maintenance $=$ & $\mathrm{M}_{1+2}$ & $\mathrm{M}_{1+2}$ & $\mathrm{M}_{1+2}$ & $\mathrm{M}_{2}$ & $\mathrm{M}_{2}$ & $\mathrm{M}_{0}$ \\
\hline $\bar{c}_{\min }=$ & 0.08977 & 0.13875 & 0.09315 & 0.11371 & 0.20811 & {$[0.05]$} \\
$N_{\text {tol }}=$ & 2871.82 & 2843.04 & 2705.25 & 2927.14 & 2927.14 & $\infty$ \\
$n_{2}=$ & 6 & 1 & - & - & - & - \\
$n_{1}=$ & 17 & 14 & 9 & 2 & 2 & - \\
\hline
\end{tabular}

(h) $\left(c_{n c}, c_{i n v, 1}, c_{i n v, 2}, c_{m, 1}, c_{m, 2}\right)=(120,5,10,11,250)$ under $\mathrm{M}_{2}$ and $=(120,5,10,11+$ $\Delta, 250+\Delta)$ under $\mathrm{M}_{1+2}$, where $\Delta=400$.

\begin{tabular}{|r|cc|lcc|c|}
\hline Investigation $=$ & $\mathrm{I}_{1+2}$ & $\mathrm{I}_{0}$ & $\mathrm{I}_{2}$ & $\mathrm{I}_{2}$ & $\mathrm{I}_{0}$ & $\mathrm{I}_{0}$ \\
Maintenance $=$ & $\mathrm{M}_{1+2}$ & $\mathrm{M}_{1+2}$ & $\mathrm{M}_{1+2}$ & $\mathrm{M}_{2}$ & $\mathrm{M}_{2}$ & $\mathrm{M}_{0}$ \\
\hline $\bar{c}_{\text {min }}=$ & 2.11196 & 2.73581 & 2.08753 & {$[1.98957]$} & 2.25668 & 6 \\
$N_{\text {tol }}=$ & 2621.72 & 2824.78 & 2627.87 & 2644.92 & 2846.52 & $\infty$ \\
$n_{2}=$ & 27 & 3 & - & - & - & - \\
$n_{1}=$ & 28 & 4 & 25 & 19 & 3 & - \\
\hline
\end{tabular}

\section{Discussion.}

In this paper, the authors apply the concept of cumulative count of conforming chart (CCC chart) to develop inspection and maintenance plans for production systems where an item produced can be classified as either conforming or nonconforming. All products items produced are inspected, and the number of items inspected until a nonconforming item is observed is used as an indicator for system monitoring. When a nonconforming item is observed, either minor investigation, major investigation or no investigation on the system may be carried out, and subsequently, either minor maintenance, major maintenance or no maintenance on the system may be carried out. The author derived analytic expressions of the probabilities for different cases to occur, expected numbers of signals, expected numbers of investigations and maintenance actions. With these results, optimized investigation and maintenance strategies are obtained numerically from an economic approach.

This paper assumes that minor deterioration of the system can be detected by minor 
investigation, and major deterioration of the system can only be detected only by major investigation, which is illustrated by an example on maintenance of a bus fleet. In reality, there is another situation where minor deterioration in the system is well-hidden and can be detected only by major inspection, whereas major deterioration is conspicuous and can be detected by minor inspection. Separate models can be established for this situation, and optimized investigation and maintenance strategies can be obtained.

This paper considers the case when system has three discrete states $\mathrm{S}_{0}, \mathrm{~S}_{1}, \mathrm{~S}_{2}$ and changes from state $\mathrm{S}_{\gamma}$ to state $\mathrm{S}_{\gamma+1}$ with probability $\pi_{\gamma, \gamma+1}(\gamma=0,1)$. This assumption has been used in the literature (for example, in Das and Jain (1997), Das et al (1996), Lorenzen and Vance (1986)) for the purpose of economic design of control charts. Separate studies can be carried out for the situation when deterioration of the system is continuous and the system has continuous states (see for example, Liao et al (2006)).

In practice, due to cost constraint or operational constraints, maintenance may not be carried out immediately even when investigations show that the system is in an early state of deterioration (Christer (1999), Liao et al (2008)). Signals on the control chart indicating minor deterioration of the system may be used an warnings, and actions will be taken only when there are signals showing more serious deterioration.

Acknowledgement. This research is supported by a CERG grant from Research Grants Council of the Government of Hong Kong SAR. The authors would like to express their thanks to the reviewers and their editor for their valuable suggestions that significantly improve the presentation of this paper.

\section{References.}

[1] Calvin, T.W. (1983) Quality control techniques for 'zero-defects'. IEEE Transac- 
tions on Components, Hybrid and Manufacturing Technology, CHMT-6(3), 323328.

[2] Cassady, C.R., Bowen, R.O., Liew, L., Pohl, E.A. (2000) Preventive maintenance and statistical process control: a preliminary investigation, IIE Transactions, 32(6), $471-478$.

[3] Chan, L.Y. (2000) Control charts for monitoring low-defect Processes. The Eighth International Conference on Manufacturing Engineering, ICME 2000, 27-30 August 2000, Sydney, Australia, P07A.02 (in CD-ROM).

[4] Chan, L.Y., Xie, M. and Goh, T.N. (1997) Two-stage control charts for high yield processes. International Journal of Reliability, Quality and Safety Engineering, 4(2), $149-165$.

[5] Chan, L.Y., Xie, M. and Goh, T.N. (2000) Cumulative quantity charts for monitoring production processes. International Journal of Production Research, 38(2), 397-408.

[6] Chan, L.Y., Lin, D.K.J., Xie, M. and Goh, T.N. (2002) Cumulative probability control charts for geometric and exponential process characteristics. International Journal of Production Research, 40(1), 133-150.

[7] Chan, L.-Y. (2003) Design of inspection and maintenance models based on the CCCchart Proceedings of the Annual Reliability and Maintainability Symposium, pp. 7481.

[8] Christer, A.H., (1999) Developments in delay time analysis for modeling plant maintenance, Journal of the Operational Research Society, 50, 1120-1137.

[9] Das, T., Jain, V. and Gosavi, A. (1997) Economic design of dual-sampling-interval policies for $\bar{X}$ charts with and without run rules. IIE Transactions, 29(6), 497-506.

[10] Das, T. and Jain, V. (1997) An economic design model for $\bar{X}$ charts with random sampling policies. IIE Transactions, 29(6), 507-518. 
[11] Goh, T. N. (1987) A control chart for very high yield processes. Quality Assurance, 13(1), 18-22.

[12] Gordon, G.R. and Weindling, J.I. (1975) A cost method for economic design of warning limit control chart schemes. American Institute of Industrial Engineers Transactions, 7, 319-329.

[13] Liao, H., Elsayed, E.A., Chan, L.Y. (2006) Maintenance of continuously monitored degrading systems, European Journal of Operational Research, 175, 821-835.

[14 Liao, H., Chan, L.Y., Elsayed, E.A. (2008) Maintenance of continuous degrading units considering random service delays, submitted.

[15] Lorenzen, T.J. and Vance, L.C. (1986) The economic design of control charts: A unified approach. Technometrics, 28(1), 3-10.

[16] Montgomery, D.C. (1980) The economic design of control charts: a review and literature survey. Journal of Quality Technology, 12(2), 75-87.

[17] Montgomery, D.C., (2001), Introduction to Statistical Quality Control. , John Wiley and Sons, New York.

[18] Page, E.S. (1955) Control charts with warning limits. Biometrika, 42, 243-257.

[19] Ranjan, P., Xie, M., Goh, T.N. (2003) Optimal control limits for CCC charts in the presence of inspection errors. Quality and Reliability Engineering International (2), pp. 149-160.

[20] Tagaras, G., Lee, H.L. (1988) Economic design of control charts with different control limits for different assignable causes. Management Science, 34(11), 1347-1366.

[21] Tagaras, G., Lee, H.L. (1989) Approximate semi-economic design of control charts with multiple control limits, Naval Research Logistics, 36, 337-353.

[22] Tagaras, G. (1998) A survey of recent developments in the design of adaptive control charts. Journal of Quality Technology, 30(3), 212-231. 
[23] Weindling, J.I., Littauer, S.B., de Oliveirva, J.T. (1970) Mean action time of the $\bar{X}$-control chart with warning limits. Journal of Quality Technology, 2(2), 79-85.

[24] Woodall, W.H. (1997) Control charts based on attribute data: bibliography and review. Journal of Quality Technology, 29(2), 172-183.

\section{Appendix.}

To find the expected number of items inspected and the expected number of each of type of signal in different cases, we need to find closed-form expressions of $I_{0}, I_{1}, \ldots, J M N_{2}$. The following identities will be used, in which $i, j, k, u, w \geq 1$ are integers, $u<w, a \neq 1$, $b \neq 1$ and $c \neq 1$. These identities are derived using $1+u+\cdots+u^{n}=\left(1-u^{n+1}\right) /(1-u)$ and its derivative, where $u \neq 1$. Corresponding results when $a=1, b=1$ or $c=1$ can be more easily derived.

$$
\begin{aligned}
& S_{1}=\sum_{i=u}^{w} a^{i-1}=\frac{a^{u-1}-a^{w}}{1-a} . \\
& S_{11}=\sum_{i=u}^{w} i a^{i-1}=\frac{a^{u}-a^{w+1}}{(1-a)^{2}}+\frac{u a^{u-1}-(w+1) a^{w}}{(1-a)}=\frac{a S_{1}}{1-a}+\frac{u a^{u-1}-(w+1) a^{w}}{1-a} \\
& =\frac{a^{u-1}(a+u-a u)-a^{w}(a+w-a w)}{(1-a)^{2}} . \\
& S_{2}=\sum_{i+j=u}^{w} a^{i-1} b^{j-1} \quad(a \neq b), \\
& =\left\{\begin{array}{l}
\frac{a^{u-1}-a^{w}}{(1-a)(a-b)}-\frac{b^{u-1}-b^{w}}{(1-b)(a-b)}, \\
\frac{(u-1) a^{u-2}-(u-2) a^{u-1}-w a^{w-1}+(w-1) a^{w}}{(1-a)^{2}}, \quad(a=b) . \\
S_{22}=\sum_{i+j=u}^{w}(i+j) a^{i-1} b^{j-1}=\left(\frac{1}{1-a}+\frac{1}{1-b}\right) \times S_{2}+\frac{1}{(1-a)(1-b)} \times\left[Q_{22}\right], \text { where } \\
{\left[Q_{22}\right]=\left\{\begin{array}{l}
\left(b^{u-1}-a^{u-1}\right)+(u-1)\left[(1-b) a^{u-1}-(1-a) b^{u-1}\right] \\
-\frac{\left(b^{w}-a^{w}\right)+w\left[(1-b) a^{w}-(1-a) b^{w}\right]}{a-b}, \\
-(u-1) a^{u-2}+(u-1) a^{u-2}[(u-1)-(u-2) a] \\
+w a^{w-1}-w a^{w-1}[w-(w-1) a],
\end{array} \quad(a \neq b),\right.}
\end{array}\right.
\end{aligned}
$$




$$
\begin{aligned}
& S_{3}=\sum_{i+j+k=u}^{w} a^{i-1} b^{j-1} c^{k-1} \\
& = \begin{cases}\frac{\left(a^{u-1}-a^{w}\right)}{(1-a)(a-b)(a-c)}-\frac{\left(b^{u-1}-b^{w}\right)}{(1-b)(a-b)(b-c)}+\frac{\left(c^{u-1}-c^{w}\right)}{(1-c)(a-c)(b-c)}, & (a \neq b \neq c) \\
\frac{\left(a^{u-1}-a^{w}\right)}{(1-a)(a-b)^{2}}-\frac{a\left((u-1) b^{u-2}-w b^{w-1}\right)}{(1-b)^{2}(a-b)^{2}} & (a \neq b=c) \\
+\frac{(1+a)\left((u-2) b^{u-1}-(w-1) b^{w}\right)}{(1-b)^{2}(a-b)^{2}}-\frac{(u-3) b^{u}-(w-2) b^{w+1}}{(1-b)^{2}(a-b)^{2}}, & (a=b=c) \\
\frac{a^{u-1}-a^{w}}{(1-a)^{3}}+\frac{(u-1) a^{u-2}-w a^{w-1}}{(1-a)^{2}}+\frac{(u-1)(u-2) a^{u-3}-w(w-1) a^{w-2}}{2(1-a)}, & \end{cases}
\end{aligned}
$$

$$
\begin{aligned}
& S_{33}=\sum_{i+j+k=u}^{w}(i+j+k) a^{i-1} b^{j-1} c^{k-1} \\
& =\left(\frac{1}{1-a}+\frac{1}{1-b}+\frac{1}{1-c}-1\right) \times S_{3}+\frac{1}{(1-a)(1-b)(1-c)} \times\left[Q_{33}\right] \text {, where } \\
& \int \frac{(1-b)(1-c)\left[(u-1) a^{u-1}-w a^{w}\right]+(-1+b c)\left[a^{u-1}-a^{w}\right]}{(a-b)(a-c)} \\
& -\frac{(1-a)(1-c)\left[(u-1) b^{u-1}-w b^{w}\right]+(-1+a c)\left[b^{u-1}-b^{w}\right]}{(a-b)(b-c)} \\
& +\frac{(1-a)(1-b)\left[(u-1) c^{u-1}-w c^{w}\right]+(-1+a b)\left[c^{u-1}-c^{w}\right]}{(a-c)(b-c)}, \quad(a \neq b \neq c), \\
& \frac{(1-b)^{2}\left((u-1) a^{u-1}-w a^{w}\right)+\left(-1+b^{2}\right)\left(a^{u-1}-a^{w}\right)}{(a-b)^{2}} \\
& +\frac{-(1-a)\left((u-1)\left[(u-3) b^{u}-(u-2)(1+a) b^{u-1}+a(u-1) b^{u-2}\right]\right)}{(a-b)^{2}} \\
& {\left[Q_{33}\right]=\left\{\begin{array}{l}
(1-a)\left(w\left[(w-2) b^{w+1}-(w-1)(1+a) b^{w}+a w b^{w-1}\right]\right) \\
(a-b)^{2}
\end{array}\right.} \\
& +\frac{a\left((u-3) b^{u}-(w-2) b^{w+1}\right)}{(a-b)^{2}}+\frac{-\left(1+a^{2}\right)\left((u-2) b^{u-1}-(w-1) b^{w}\right)}{(a-b)^{2}} \\
& +\frac{a\left((u-3) b^{u}-(w-2) b^{w+1}\right)}{(a-b)^{2}}, \quad \quad(a \neq b=c) \\
& =\frac{3\left(a^{u}-a^{w+1}\right)}{(1-a)^{4}}+\frac{3\left(u a^{u-1}-(w+1) a^{w}\right)}{(1-a)^{3}}+\frac{3\left(u(u-1) a^{u-2}-(w+1) w a^{w-1}\right)}{2(1-a)^{2}} \\
& +\frac{\left.u(u-1)(u-2) a^{u-3}-(w+1) w(w-1) a^{w-2}\right)}{2(1-a)}, \quad(a=b=c) \text {. }
\end{aligned}
$$

